PPPL-3443

PPPL-3443

UC-70

Heatpulse Propagation Studies on DIII-D and TFTR

by

E. Fredrickson, M.E. Austin, R. Groebner, J. Manickam,

B. Rice, G. Schmidt, and R. Snider

April 2000
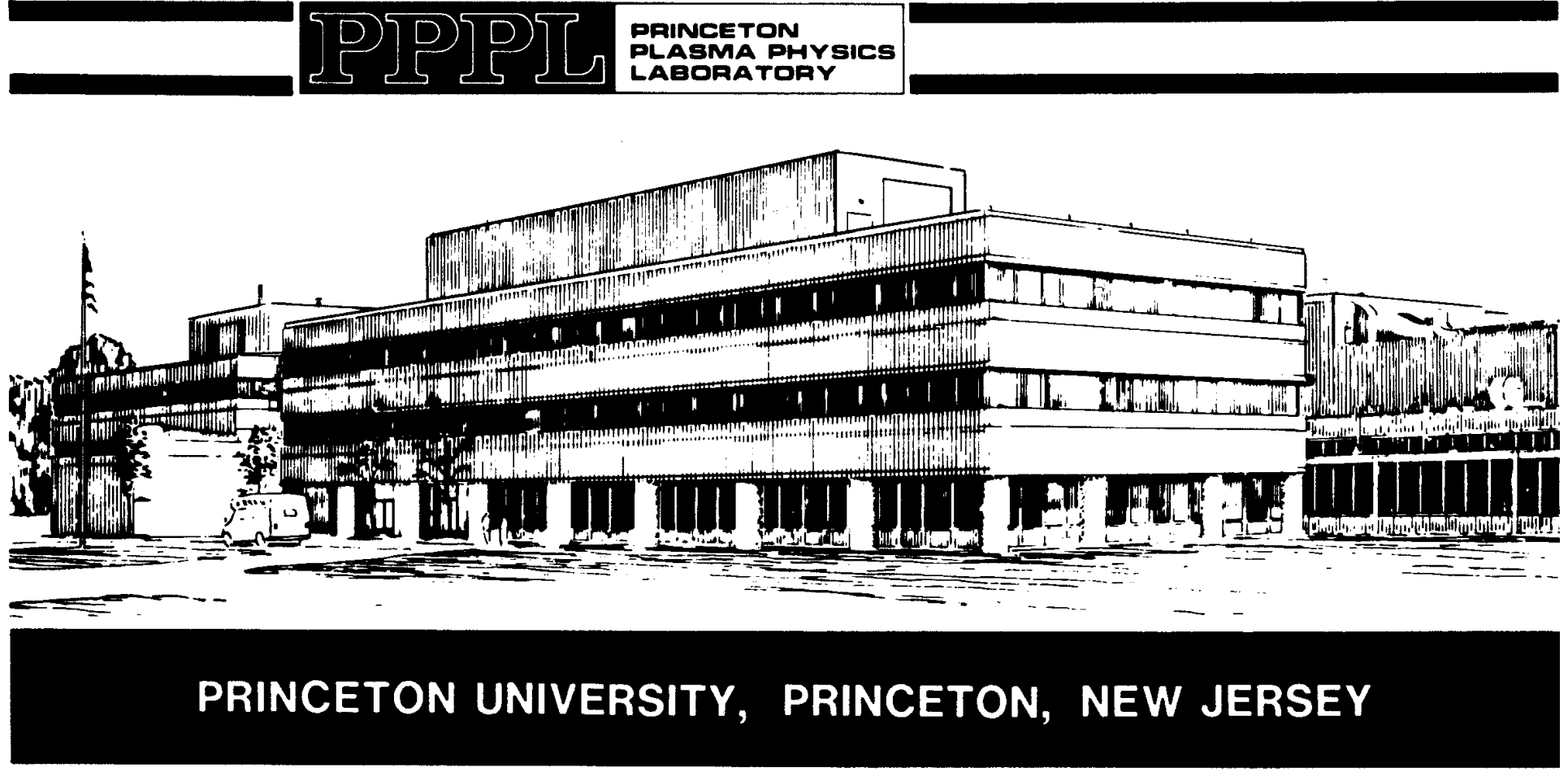


\section{PPPL Reports Disclaimer}

This report was prepared as an account of work sponsored by an agency of the United States Government. Neither the United States Government nor any agency thereof, nor any of their employees, makes any warranty, express or implied, or assumes any legal liability or responsibility for the accuracy, completeness, or usefulness of any information, apparatus, product, or process disclosed, or represents that its use would not infringe privately owned rights. Reference herein to any specific commercial product, process, or service by trade name, trademark, manufacturer, or otherwise, does not necessarily constitute or imply its endorsement, recommendation, or favoring by the United States Government or any agency thereof. The views and opinions of authors expressed herein do not necessarily state or reflect those of the United States Government or any agency thereof.

\section{Availability}

This report is posted on the U.S. Department of Energy's Princeton Plasma Physics Laboratory Publications and Reports web site in Calendar Year 2000. The home page for PPPL Reports and Publications is: http://www.pppl.gov/pub_report/

DOE and DOE Contractors can obtain copies of this report from:

U.S. Department of Energy

Office of Scientific and Technical Information

DOE Technical Information Services (DTIS)

P.O. Box 62

Oak Ridge, TN 37831

Telephone: (865) 576-8401

Fax: (865) 576-5728

Email: reports@adonis.osti.gov

This report is publicly available from:

National Technical Information Service

U.S. Department of Commerce

5285 Port Royal Road

Springfield, VA 22161

Telephone: 1-800-553-6847 or

(703) $605-6000$

Fax: (703) 321-8547

Internet: http://www.ntis.gov/ordering.htm 


\title{
Heatpulse Propagation Studies on DIII-D and TFTR
}

\author{
E. Fredrickson ${ }^{1}$, M.E. Austin ${ }^{2}$, R. Groebner ${ }^{3}$, \\ J. Manickam ${ }^{1}$, B. Rice ${ }^{4}$, G. Schmidt ${ }^{1}$, R. Snider ${ }^{3}$ \\ 1. Princeton Plasma Physics Laboratory, Princeton, New Jersey \\ 2. University of Texas, Austin, Texas \\ 3. General Atomics, La Jolla, California \\ 4. Lawrence Livermore National Laboratory, Livermore, California \\ Present address: Xenogen Corp., Alameda, California
}

Sawtooth phenomena have been studied on DIII-D and TFTR. In the experiments, with high power neutral beam injection the sawtooth characteristics were studied with fast electron temperature (ECE) and soft x-ray diagnostics. A strong ballistic electron heat pulse is found on DIII-D, stronger than was previously reported on TFTR. Evidence is presented in this paper that the ballistic effect is related to the sawtooth precursor. Fast, $2 \mathrm{msec}$ interval, measurements on DIII-D were made of the ion temperature evolution following the sawtooth to document the ion heat pulse characteristics. It is found that the ion heat pulse does not exhibit the very fast, "ballistic" behavior seen for the electrons. Further, both the electron and ion heat pulses from partial sawtooth crashes and similar events are seen to propagate at speeds close to those expected from the power balance calculations of the thermal diffusivities. These results suggest that the fast sawtooth induced heat pulse propagation is not a feature of non-linear transport models, but that MHD events can have a strong effect on thermal transport. 


\section{INTRODUCTION}

The sawtooth instability was discovered on the ST tokamak [1] and a heuristic model of the instability consistent with the limited experimental data of that time was proposed by Kadomtsev [2]. Subsequently inconsistencies between the Kadomtsev model of the sawtooth and experimental measurements of the sawtooth instability were found $[3,4]$. Presently, no complete model of the sawtooth predicts the wide range of sawtooth behavior seen in many tokamaks. This paper presents data from experiments on DIII-D and the Tokamak Fusion Test Reactor (TFTR) related to the sawtooth instability.

The experiment on DIII-D was carried out as represented in Figure 1a. There is an early period of Ion Cyclotron Range of Frequency (ICRF) heating between $\approx 1.8-3.3 \mathrm{~s}$ followed by a high power Neutral Beam Injection (NBI) heating phase from approximately 3.9 - $4.6 \mathrm{~s}$. Some NBI was used throughout for diagnostic purposes and, as discussed in Ref. 5, to provide a seed population of energetic deuterium ions for $4^{\text {th }}$ harmonic ICRF heating. This paper will discuss predominantly sawtooth related phenomena during the high power NBI phase. The object of this part of the experiment was to study sawtooth stabilization utilizing NBI. The ICRF phase of the experiment was a study of sawtooth stabilization from ICRF-generated tail ions [5]. The sawtooth period was increased to as much as $250 \mathrm{msec}$ with either NBI or ICRF, but full suppression of the sawteeth, as was commonly observed on TFTR [6], was not seen.

The TFTR data presented here was taken during a limiter conditioning sequence to achieve high performance D-T plasmas. The evolution of the plasma is shown in Fig. 1b. Several high current, moderately high NBI power shots were taken to remove Deuterium from the limiter. The reduction in Deuterium recycling at the plasma edge results in higher edge temperatures and improved global confinement. Concurrent with this improved performance, the sawtooth period lengthens until the sawteeth are stabilized for the period of the NBI injection. The sawteeth discussed in this paper come from shots before good limiter conditioning was achieved, but which have reasonably good performance, with $\tau_{\mathrm{E}} \approx$ $1.5 \tau_{\mathrm{E}}^{\mathrm{L} \text {-mode }}$.

In Fig. 2 representative q-profiles for these DIII-D and TFTR plasmas are compared. The q-profile for the DIII-D shot was measured with a Motional Stark Effect (MSE) system $[7,8]$. The q-profile for the TFTR plasma is calculated by solving the resistive current diffusion equation with the TRANSP code [9]. For these shots the $\mathrm{q}_{95}$ (edge q) and aspect ratio of the $\mathrm{q}=1$ surface, $\mathrm{r}_{\mathrm{q}=1} / \mathrm{R}_{0}$, are comparable for the DIII-D and TFTR plasmas. The $\mathrm{q}$ 
profile evolution through the sawtooth crash on DIII-D differs from TFTR in that following the sawtooth crash, the measurements of the current profile on DIII-D show that the $q(0)$ has risen to approximately unity, consistent with previous findings [10]. Previous experiments on TFTR where the q profile was measured with an MSE diagnostic had found that the q remained well below unity following the sawtooth [11].

The principal diagnostic for studying the sawtooth precursor was an Electron Cyclotron Emission (ECE) radiometer on DIII-D and two ECE Grating Polychromators (GPCs) on TFTR [12]. The characteristics of the DIII-D sawtooth precursor appear very similar to what was found in previous studies on the TFTR tokamak [13-16]. A flat spot in the electron temperature profile, which is assumed to indicate the presence of an $m=1, n=1$ island, is seen to grow in the final 100's of $\mu$ s before the sawtooth crash. The growth of the island, as deduced from the ECE measurements, appears consistent with a Kadomtsevtype reconnection on both TFTR and DIII-D. That is, the island appears to grow until it encompasses the entire plasma core resulting in the sawtooth crash.

The relaxation of the electron and ion temperature profiles towards equilibrium following the sawtooth crash was studied using the fast ECE diagnostics and on DIII-D with the fast $(2 \mathrm{~ms})$ charge exchange recombination (CER) measurements of the ion temperature profile. It was found that the sawtooth induced electron heat pulse propagation showed an even stronger ballistic character on DIII-D than was observed on TFTR $[17,18]$, demonstrating that the ballistic heat pulse first observed on TFTR is not unique to that machine. Of particular interest, the detailed measurements of the sawtooth induced ion temperature heat pulse suggest that the ion heat pulse is not strongly ballistic, supporting earlier conjectures that the ballistic character of the electron heat pulse is due to weak magnetic stochasticity[18]. The propagation of heat pulses from partial sawteeth and fishbones is used to help to clarify the origin of the transport enhancement responsible for the ballistic heat pulse.

\section{PHYSICS OF THE SAWTOOTH CRASH}

The Kadomtsev model of the sawtooth instability is that the $m=1, n=1$ tearing mode grows to completely reconnect the core of the plasma. The Kadomtsev model further predicts that the rotational transform parameter, $\mathrm{q}$, would be raised above unity within the reconnection radius. Detailed studies of the sawtooth phenomena using fast ( $2 \mu$ s sample time) ECE grating polychromators for the electron temperature profile diagnostic and soft $\mathrm{x}$-ray pinhole cameras on TFTR found evidence of the predicted $(1,1)$ tearing mode sawtooth precursor. However, measurements on TEXTOR [3] and TFTR [11] found that $q(0)$ 
remained below unity following the sawtooth crash, in contradiction to the prediction of the Kadomtsev model. It will be shown below that the sawtooth precursor structure seen on DIII-D is very similar to that on TFTR, suggesting that the precursor instability is the same. However, the MSE measurements of the q profile find that $q(0)$ does jump to unity during the sawtooth reconnection (c.f. Fig. 2). There is as yet no explanation for this difference in behavior.

Other than the behavior of $q(0)$, many aspects of the sawtooth instability appear similar in TFTR and DIII-D. In the fast measurements of the electron temperature profile it can be seen that for both machines the precursors have a "ballooning" $\mathrm{m}=1$ structure. Initially, the precursor is kink-like (small island) with a slow growth rate, followed by a period during which the island grows rapidly. The island growth rates can vary over the range from less than $10^{3} / \mathrm{s}$ to greater than $10^{4} / \mathrm{s}$. The sawtooth precursors growth rate has been discussed extensively in previous papers [e.g., Refs. 14 and 18] and will not be discussed further here.

A contour plot of the electron temperature through a typical sawtooth on TFTR as measured with a $2^{\text {nd }}$ harmonic grating polychromator is shown in Figure 3 . Temperature contours are drawn every $0.5 \mathrm{keV}$. The sawtooth precursor island begins to grow 700 $\mu$ sec prior to the crash. As the precursor island becomes larger, the $m>1$ components of the mode, i.e., beyond the $\mathrm{q}=1$ surface, become visible in the ECE electron temperature data.

The data from Mirnov coils, which measure predominantly the $\mathrm{m} \approx \mathrm{q}(\mathrm{a})$ components, shows a qualitatively similar behavior. The precursor amplitude before the rapid island growth starts is about $1 \mathrm{G}$ and increases with the growth of the island until it reaches about $3 \mathrm{G}$ in amplitude at the time of the crash. The scaling of the magnetic fluctuation amplitude with island size is complex, as shown in Fig. 4. Initially, the magnetic fluctuation level scales roughly with the square root of the island width; recall that for tearing modes the magnetic fluctuation level scales as the square of the island width. During the final, rapid island growth, the magnetic fluctuation level increases even more slowly with island width. This result implies that the poloidal structure of the $n=1$ sawtooth precursor is changing as the island grows.

In Figure 5 is shown a sawtooth in a plasma with similar parameters to that in Figure 3. Here the island growth starts approximately $100 \mu$ s before the crash. In this example, the precursor oscillations are essentially undetectable in the ECE data beyond the $q=1$ surface. The phase of the $n=1$ mode is such that the $x$-point is on the inboard side during the final period of rapid island growth, thus ECE system could not measure the amplitude of the 
$\mathrm{m}>1$ components. However, the Mirnov loop data show that the magnetic fluctuation grows from $\approx 1 \mathrm{G}$ to $3 \mathrm{G}$ in the final period of rotation.

There is a further observation, which is of interest, in the TFTR data. In the final phase of the precursor growth for some sawteeth there is evidence for a localized, intermediate $n$ ballooning mode. This is very similar to the observation of coupled $n=1$ kink and intermediate $\mathrm{n}$ ballooning modes believed responsible for beta limit disruptions on TFTR $[19,20]$. An example, clearer than most, is shown in Figure 6. This is a contour plot of the electron temperature as measured at $2 \mu$ s sampling interval. The final two oscillations of the $n=1$ sawtooth precursor are shown. The last oscillation before the sawtooth crash was coincidentally towards the outboard side at the toroidal location of the GPC. This enabled the observation of the ballooning mode, seen as two oscillations of a higher frequency mode just before the crash on the outboard side.

An example of a sawtooth precursor on DIII-D as measured with the heterodyne radiometer is shown in Figure 7. Contours are also shown every $0.5 \mathrm{keV}$ and the digitization rate is $100 \mathrm{kHz}$. The characteristic precursor frequency (plasma rotation rate) is $15 \mathrm{kHz}$, vs. the $5 \mathrm{kHz}$ in the TFTR example. The general behavior is similar to that on TFTR, with the precursor present for many msec at nearly constant amplitude, followed by a phase of rapid growth. In this case the rapid growth begins $300 \mu$ s before the sawtooth crash. The coupling to poloidal harmonics is stronger, seemingly a general characteristic of DIII-D sawtooth precursors.

The time dependent electron temperature profile data can be mapped to a 2-D crosssectional image, even for shaped plasmas, with some reasonable assumptions about the mode rotation and helicity [21]. The reconstruction begins with the ECE data mapped to minor radius using, for DIII-D, EFIT. A reconstruction of the data shown in Figure 8a can be seen in Figure $8 b$. The reconstruction, done in $\rho-\theta$ space is mapped to "real-space" assuming a constant elongation of 1.24 in the core, as determined from an EFIT equilibrium reconstruction. The mapping does not include a Shafranov shift nor higher order moments such as triangularity or squareness (these last two are small near the axis). The characteristic MHD frequency in DIII-D is relatively close to the instrumental bandwidth, which precludes a very detailed reconstruction of the sawtooth precursor. However, in this data set, it is possible to see that the sawtooth precursors have the classic "cold island" structure typically seen in TFTR sawtooth precursors [13,14].

\section{Transport studies using perturbation methods}


Studies of sawtooth induced heat pulse propagation were originally undertaken as a method for direct measurement of energy transport coefficients in tokamak plasmas [22-24]. The Kadomtsev reconnection model of the sawtooth crash predicts that the change in the electron temperature profile through the sawtooth crash is constrained to be within the reconnection radius. Thus it could be assumed that the thermal transport after the sawtooth crash and outside the reconnection radius was governed by a simple diffusion equation. Initial studies of sawtooth induced heat pulses on TFTR found that they propagated much faster than was consistent with the thermal diffusivity derived from power balance calculations. Several models were proposed to explain this discrepancy, including models that the thermal diffusivity was time dependent, either explicitly as a result of MHD activity $[16,24]$ or implicitly through a non-linear dependence of the transport on local plasma properties such as $\mathrm{T}_{\mathrm{e}}$ or $\nabla \mathrm{T}_{\mathrm{e}}$ [24].

The Kadomtsev model prescribes how the plasma thermal energy is mixed between volumes within the $\mathrm{q}=1$ surface (inversion radius) and an annular volume between the $\mathrm{q}=1$ surface and the reconnection or mixing radius. As the profile is changed through a reconnection process, rather than, for example, a large increase in transport, the post crash profile need not be flat or rounded. The reconnection radius is determined by the amount of flux to be reconnected within the $\mathrm{q}=1$ surface. In hot plasmas, such as DIII-D and TFTR, there is relatively little flux diffusion into the core between sawtooth crashes so that the Kadomtsev reconnection radius is only slightly larger than the radius of the $\mathrm{q}=1$ surface. This tends to result in predictions of hollow post crash temperature profiles and on TFTR the post crash electron temperature profile was indeed often slightly hollow. The prediction for DIII-D was also that the temperature profile would be hollow, but the observed postcrash profiles were generally rounded (slightly peaked).

On TFTR, for very fast sawtooth crashes, the initial temperature perturbation was consistent with the predictions of the Kadomtsev reconnection model. However, on a very short time scale, $\approx 100 \mu$ s, the profile relaxed and the heat was moved well beyond the reconnection radius. This effect was named the "ballistic heat pulse" as it occurred more than an order of magnitude faster than expected from the power balance calculations of the thermal diffusivity. The radial and temporal extent of the enhancement in thermal diffusivity could not be accurately determined from the data and it was concluded that sawtooth induced heat pulses were unreliable indicators of "equilibrium" transport coefficients [24,25]. The "ballistic" contribution to the electron heat pulse was similar or stronger in the DIII-D data presented here than was seen on TFTR. 
The discovery of the ballistic component of sawtooth induced heat pulses made the use of these measurements impractical as a direct local measurement of the diffusivity. However, studies of sawtooth induced heat pulses are still of interest for their implications regarding the mechanism of the sawtooth crash and the enhancement in heat transport. The ballistic heat pulse provides a window on the, as yet, not understood physical mechanism of the sawtooth crash itself. And while the heat pulse may not be used as an independent measure of the equilibrium transport, it does challenge present models of thermal transport; even the most strongly non-linear models of thermal transport do not predict the observed strength of the ballistic response. Further, if the mechanism responsible for the enhancement in transport during the ballistic response were operating at even a fraction of its apparent capability at all times, it could account for all of the anomalous electron thermal transport.

\section{IIIa. Heat Pulse Simulations}

The clearest method of determining whether heat pulses have a ballistic character is by comparison of the measured heat pulses to heat pulse simulations. The simulations presented here for this purpose are either initial value simulations where a 1-D single fluid heat transport code calculates the evolution of the temperature profile with periodic "sawteeth" or boundary value perturbation solutions. The boundary value simulations use the measured perturbations in the temperature, either as a time dependent radial boundary condition or by introducing a radial profile of the temperature perturbation, which is then allowed to relax in time. The initial value simulations can additionally employ non-linear transport coefficients or explicitly time dependent thermal diffusivities [25].

The code uses as input the density profile shape and the thermal diffusivity $\chi(r)$. For non-perturbative simulations the heating profile is chosen so that the temperature profile matches the measured profile. These profiles are input as multi-parameter analytical functions, which have been fit to the experimental data, or in the case of the diffusivity, the output from the TRANSP code. The TRANSP code uses as input various measured plasma profiles as well as information from the NBI heating system and equilibrium data from the EFIT code. From this data it calculates the evolution in time of the electron and ion transport co-efficients.

\section{IIIb. Sawtooth Induced Electron Heat Pulse Propagation}

The simulations of heat pulse propagation begin with some assumptions about the nature of the sawtooth event. The simplest model is that the sawtooth follows the 
Kadomtsev prescription for redistribution of the heat and deviations from those predictions are attributed to anomalous transport, i.e., the ballistic character of the heat pulse. The ballistic nature can be seen Fig. 9a where the measured $\mathrm{T}_{\mathrm{e}}$ profiles from before and after the sawtooth crash (shown in Fig. 7) are compared to a simulation of the post-crash profile using the Kadomtsev model. The pre-crash q-profile as measured with the MSE diagnostic is used in the simulated reconnection, but the density was assumed flat in the absence of a measured density profile in the core; a peaked density would have resulted in a more hollow post crash profile. The measured post crash profile is taken very near the end of the reconnection phase. The inversion, or $\mathrm{q}=1$ radius, and the calculated reconnection radius are indicated.

During the short time of the reconnection phase, there is substantial propagation of thermal energy well beyond the reconnection radius. In this example the reconnection phase, defined as the period of rapid growth of the island, takes less than $200 \mu \mathrm{s}$. In Fig. $9 \mathrm{~b}$ is shown a simulation of a sawtooth-like event, instantaneous in time, and post crash profiles immediately after and $200 \mu \mathrm{sec}$ after the crash. Even $200 \mu \mathrm{sec}$ after the crash there is a significant temperature rise only within a few $\mathrm{cm}$ of the mixing radius. The rapid propagation of heat beyond the mixing radius seen in Fig. 8a is postulated to be the result of a large enhancement in diffusion around the $\mathrm{q}=1$ surface during and for a brief period following the sawtooth crash. It should be noted that a sampling time at least as short as 10 $\mu \mathrm{s}$ in the temperature profile measurement is necessary for accurate determination of the time interval of the reconnection and the determination of the diffusive nature of the ballistic response.

While the "diffusive" transport of heat to well beyond the mixing radius in $100-200$ $\mu$ s is qualitative evidence of the large and transient enhancement in $\chi$, it is instructive to make quantitative comparisons with heat pulse simulations. In figures 10-12 are shown three simulations of heat pulses compared to the measured heat pulses. The radii of the ECE channels are indicated on the figure together with the range of the "auto-scaled" temperature perturbations. In the first simulation, Fig. 10, the full electron temperature profile evolution in time is calculated using $\chi \approx \chi^{\mathrm{PB}}$, with artificial "sawteeth" imposed on the profile at regular intervals. The sawteeth are simulated by redistributing the heat within a specified mixing radius to give a flat, or, as in this case, hollow post crash profile to approximately match the expected post crash profile as predicted from the Kadomtsev model (Fig. 9b). This simulation gives the slowest and weakest electron response. The 
simulation is clearly not consistent with the measurements as a significant temperature rise is not seen at $r=0.39 \mathrm{~m}$ until many $\mathrm{ms}$ after the crash, and even then with much smaller amplitude.

The simulation of the thermal reconnection, and even the subsequent heat transport within the mixing radius may be incorrect given the uncertainties within the Kadomtsev sawtooth model. These effects can be compensated for by using as a boundary condition the measured temperature perturbation at a radius greater than the mixing radius in the heat pulse simulation. Note that this approach would also compensate for any "ballistic" effect localized within the radius of the measured temperature perturbation. The simulation in Fig. 11 is done in this manner by using the perturbed electron temperature at $r=0.34 \mathrm{~m}$ and still using $\chi_{\mathrm{e}} \approx \chi_{\mathrm{e}}{ }^{\mathrm{PB}}$. The simulated heat pulse is much stronger and faster than the first simulation, but still much weaker and slower than the actual heat pulse, demonstrating that either the "ballistic effect" or non-linear transport exists beyond this radius.

It is instructive to examine one further simulation. The effect of non-linear $\chi$ 's can be simulated with a linearized driven boundary value simulation working only with the perturbations in $\mathrm{T}_{\mathrm{e}}$ and using a larger $\chi$ than found in the power balance calculation [24,25]. In the simulation shown in Fig. 12 the $\chi_{\mathrm{e}}^{\mathrm{PB}}$ is enhanced so as to match the timeto-peak of the temperature perturbation at the largest radius, $0.50 \mathrm{~m}$. In this case, even with an enhancement of 20 (representing an extremely non-linear $\nabla \mathrm{T}$ dependence in $\chi$ ) the simulation fails to reasonably match the measured heat pulse shapes at the intermediate radii. For example, at $r=0.39 \mathrm{~m}$ the rise of the simulated pulse is much weaker than in the measured heat pulse.

For comparison a TFTR heat pulse is simulated in Fig. 13. To match the time-to-peak at a minor radius of $0.75 \mathrm{~m}(\mathrm{r} / \mathrm{a} \approx 0.86)$ required an enhancement in $\chi_{\mathrm{e}}$ over $\chi_{\mathrm{e}}{ }^{\mathrm{PB}}$ of only 6 . (To match the time evolution exactly requires localized, instantaneous enhancements much larger than this.) This level of enhancement is typical for matching the time-to-peak of heat pulses on TFTR, thus by this measure the DIII-D ballistic response is $\approx 3$ times stronger than in TFTR. More extensive discussion of non-linear and ballistic heat pulse simulations and the scaling of this effect on TFTR can be found in Ref. 25.

To find the radial extent of the ballistic effect, the simulations can be started at larger and larger minor radii. However, the pulses become smoother as they move out, and the change in shape to the furthest out pulse becomes less, so that information on the temporal and spatial dependence of $\chi$ becomes less precise. Simulations can also be done using the 
radial temperature perturbation profile beginning at various times after the crash. These also show that the enhancement in $\chi$ necessary to match the data is very large and that the enhancement must persist for many milliseconds following the crash.

The onset of the "ballistic" effect starts with the onset of rapid island growth (c.f. Fig. 7) and thus is also coincident with an increase in the strength of the $m>1$ components of the $\mathrm{n}=1$ sawtooth precursor. This correlation of the enhanced diffusivity with the onset of island growth is particularly evident in the data from DIII-D shown in Figure 14. In this example, it appears that the $(1,1)$ island begins to grow from the kink at $4.7295 \mathrm{~s}$, but inexplicably stops some $300 \mu$ s later. Careful inspection of the electron temperature contours between $r=0.3$ and 0.4 m clearly show a temperature increase explicable only from transport of heat from the core. Again, for the heat to be transported on this time scale, the enhancement in the thermal diffusivity must have been substantial. During the final growth of the island, just prior to the sawtooth reconnection, the temperature increase is much more visible.

If the $\chi$-enhancement were tied to island growth, it might then be expected that sawtooth crashes with more slowly growing islands would exhibit even stronger ballistic heat pulses, as the enhancement in diffusivity would be present for a greater period of time. On the contrary, it is seen that sawteeth with very short island growth times seem to have a ballistic response that is as strong if not stronger, implying that the strength of the enhancement in diffusivity was also somehow correlated with the island growth rate.

\section{IIIc. Sawtooth induced Ion heat pulse propagation studies}

It is of interest to determine whether the ion heat pulse propagation is also strongly anomalous or approximately consistent with power balance estimates of ion thermal diffusivity. Until recently, diagnostics capable of direct measurements of the ion heat pulse were not available [26]. In this experiment measurements of the ion temperature profile were made at $2 \mathrm{msec}$ intervals with a spatial resolution of 3-5 cm using the DIII-D chargeexchange recombination (CER) system. An initial value simulation of ion heat pulses using the $\chi_{\mathrm{i}}$ determined from power balance calculations is shown in Figure 14 and compared to the measured ion temperature data. The speed and sensitivity of the CER diagnostic were sufficient to have detected a ballistic response in the ion heat transport. However, the system is only marginally capable of the detection of the weaker heat pulses propagating with the power balance $\chi_{i}$. In this simulation the ion temperature profile is approximately matched and the heat is redistributed as during a sawtooth crash to trigger a heat pulse. The 
simulated data has been smoothed with a 2 msec window to simulate the time response of the CER system. The inverted ion temperature sawtooth (heat pulse) expected between the $\mathrm{q}=1$ and mixing radii $(\mathrm{r}=0.26$ and $0.29 \mathrm{~m})$ was not detected. The simulation suggests that the inverted sawtooth would only be visible for approximately one time point and it is possible that the diagnostic is not sufficiently sensitive to detect that change. The simulated heat pulse in the region immediately outside the reconnection radius is weak, and it is difficult to conclude whether a heat pulse is present in the data.

Ion temperature heat pulses coincident with the sawtooth crash are observed at larger minor radius. They can be seen in a careful perusal of data from radii of 0.46 and $0.50 \mathrm{~m}$ in Fig. 15. The heat pulses are larger and faster than expected from the $\chi_{i}=\chi_{i}{ }^{\mathrm{PB}}$ simulation. A completely satisfactory explanation for these heat pulses has not been found, but they could be the result of a non-linear coupling between the electron and ion heat transport coefficients in this region.

\section{IIId. Heat Pulse propagation from partial sawteeth.}

Compound sawteeth are seen on both DIII-D [27] and TFTR [14,18]. Compound sawteeth have a partial reconnection in an annular region around the $\mathrm{q}=1$ surface between major sawtooth crashes. These events are referred to as partial sawteeth as the reconnection does not extend through the core. These events range from fast, off-axis reconnection-like events, to slower events more reminiscent of the collapse of an internal transport barrier. Even without the fast, partial reconnection, this event is correlated with a strong change in the core confinement. Of greater interest, the rate of heat pulse propagation for these partial sawteeth is much slower than for a sawtooth induced heat pulse, independent of whether the event was a strong, reconnection-like event or a much slower loss of core confinement.

The propagation of the ion heat pulse can be seen in Figure 16 where the ion temperature evolution at several radii, as measured with the CER diagnostic, is plotted. The rate of propagation of the heat pulse is consistent with a local diffusivity of order 5-10

$\mathrm{m}^{2} / \mathrm{sec}$. A simulation of the ion heat pulse propagation is overlaid where the diffusivity used is the power balance diffusivity. This observation of heat pulses propagating at rates consistent with the power balance thermal diffusivity suggests that the ion heat transport is not non-linear and that the rapid propagation of the sawtooth induced electron heat pulse is a characteristic of the sawtooth event itself.

A similar result is seen in the electron temperature heat pulses from partial sawtooth crashes on TFTR. The electron heat pulse propagation from two different partial sawteeth 
in similar discharges is shown in Figures 17 and 18. In Fig. 17 the heat pulse from a very slow partial crash is compared to a simulation using $\approx 2 \chi_{\mathrm{e}}{ }^{\mathrm{PB}}$. In Fig. 18 a stronger heat pulse from a much faster event is again compared to a simulation using $\chi_{\mathrm{e}} \approx 2 \chi_{\mathrm{e}}{ }^{\mathrm{PB}}$. Recall that for the sawtooth heat pulse it was necessary to enhance $\chi_{\mathrm{e}}$ by a factor of six to match the time-to-peak. These results suggest that there is a qualitative difference between the heat pulse propagation from sawtooth crashes and those resulting from other, albeit still MHD, phenomena. These results provide further evidence that the rate of sawtooth induced heat pulse propagation reflects some character of the MHD instability rather than non-linear transport in the bulk plasma.

\section{IIIe. Heat Pulse propagation from fishbone bursts.}

This disparate behavior between the propagation of sawtooth induced heat pulses and those induced by partial sawteeth suggests either that the MHD influences the electron heat transport, or, that the weaker, partial sawtooth induced, heat pulses results in their slower propagation. An even weaker heat pulse results from the "fishbone" instability [28] seen between sawtooth crashes in the TFTR plasmas. The fishbone is an $m=1, n=1$ mode driven unstable through an interaction with the fast ion tail from NBI. The mode occurs in bursts, each of which is observed to result in a drop in the core electron temperature, thereby inducing weak heat pulses. In Fig. 19 are shown the heat pulses from a series of fishbones in the same shot as the sawtooth shown in Fig. 6. The Mirnov coil signal shows the multiple fishbone bursts, with an amplitude at the plasma edge of $\approx 2 \mathrm{G}$. The individual traces of electron temperature at minor radii of $\approx 0.09 \mathrm{~m}, 0.15 \mathrm{~m}$, and $0.21 \mathrm{~m}$ show a drop in the core electron temperature through each fishbone burst. Interestingly, the enhancement in core heat transport is only present during the growth of the fishbone. Electron temperature traces at larger radii show heat pulses propagating outward. In the same figure are shown heat pulse simulations using $\chi_{\mathrm{e}} \approx 6 \chi_{\mathrm{e}}^{\mathrm{PB}}$ to match the time-to-peak at a minor radius of $0.75 \mathrm{~m}$. This is the same enhancement in $\chi_{\mathrm{e}}$ that was used to fit the much larger sawtooth induced ballistic heat pulses. The fishbone-induced heat pulse amplitude at $\mathrm{r}=0.35 \mathrm{~m}$ is $\approx 80 \mathrm{eV}$ compared to the $220 \mathrm{eV}-600 \mathrm{eV}$ amplitudes seen with the partial sawtooth heat pulses and the $>1 \mathrm{keV}$ sawtooth induced heat pulse. It can thus be concluded that the heat pulse propagation rate is more affected by other things than the heat pulse amplitude. 


\section{DISCUSSION}

It was shown above that the electron heat pulses from sawtooth crashes and from fishbone bursts propagate at many times the rate predicted from the power balance derived value of $\chi$. This behavior is best explained by a transient enhancement in the heat diffusivity. It was further shown that, in the case of the sawtooth heat pulse, this enhancement in $\chi$ began at the onset of fast growth of the island. Fast ion temperature profile measurements indicated that the ion heat pulse was not "ballistic", consistent with previous observations that the density pulse is roughly consistent with the equilibrium particle diffusivity. The observation that heat pulses from partial sawtooth events in the same plasmas propagate at rates consistent with power balance estimates of $\chi$ strongly suggests that the rapid heat transport is peculiar to the sawtooth (or fishbone) instability, rather than a feature of the bulk plasma. Further, the simulations shown in Figures 12, 13 and 19 clearly show that the enhancement due to the ballistic effect in the inferred $\chi$ extends to radii greater than twice the inversion radius, i.e., nearly to the plasma edge.

Possible causes of the rapid heat pulse propagation have been extensively investigated and discussed in previous works. Two models were proposed above to explain the fast electron heat pulse propagation. One implied a strongly non-linear electrostatic transport mechanism causing the temperature profile shape to be extremely "stiff"; any attempt to modify the gradient or gradient scale length would result in a large enhancement in transport. However, as the transport is the result of electrostatic turbulence, both the ion and electron heat transport would be affected, as well as the density transport. Experiments on TFTR showed that the sawtooth induced density pulse was much slower than the temperature pulse [29]. These results are more consistent with a model of weak magnetic stochasticity generated at the sawtooth crash, which would be expected to mainly affect electron heat transport. The parallel electron heat transport will be much faster than the parallel density transport. The parallel ion heat transport should also be slow, thus the ion heat pulse would not be expected to be greatly affected by weak magnetic stochasticity. Perpendicular ion heat transport might also be less affected if the scale lengths for the magnetic stochasticity were shorter than the ion Larmor radius.

There is some direct experimental evidence for both of these models. The $n=1$ sawtooth precursor is observed to be strongly ballooning in plasmas where a ballistic heat pulse is observed. Further, intermediate $\mathrm{n}$ ballooning modes have been observed in some cases (Fig. 6). Transient $(100 \mu \mathrm{s})$ bursts of short wavelength $\left(\mathrm{k}_{\theta} \approx 7 \mathrm{~cm}^{-1}\right)$ density fluctuations were measured with an X-mode scattering system on TFTR [30]. The bursts 
were correlated, within the spatial and temporal resolution of the scattering system $(\Delta \mathrm{z} \approx 30$ $\mathrm{cm}$ ), with the passing of the $\mathrm{x}$-point of an $\mathrm{m}=1$ island through the scattering volume of the system. These fluctuations could represent enhanced electrostatic turbulence, or turbulence during the tearing of magnetic fields leading to a weakly stochastic region.

Whether the "ballistic heat pulse" is a result of MHD activity or a consequence of strongly non-linear transport, the observation is sobering. These data clearly demonstrate that a heat transport mechanism exists which is many times more effective at transporting heat than the equilibrium transport process. If this mechanism were active at all times at only a small fraction of the efficiency exhibited during the sawtooth crash, it could entirely explain the anomalous, large electron energy transport.

\section{SUMMARY}

In this paper we have presented a comparison of sawtooth phenomenon on the DIII-D and TFTR devices. The coupling of the $n=1$ the sawtooth precursor to higher poloidal mode number were more extreme on DIII-D than on TFTR. The sawtooth precursor behavior was otherwise qualitatively very similar between the two machines. The $n=1$

precursor has an initially slow growth rate, followed by a short period (100 $\mu$ s to $1 \mathrm{~ms}$ ) during which the $m=1, n=1$ island grows rapidly. While the precursor characteristics were very similar, the striking difference between the two machines was that the sawtooth reconnection brought $\mathrm{q}(0)$ to 1 on DIII-D, but not on TFTR.

The sawtooth induced electron and ion heat pulse propagation was studied and the sawtooth induced electron heat pulse is even more strongly ballistic than on TFTR. The onset of the ballistic transport enhancement was found to coincide with the onset of fast $1 / 1$ island growth indicating that enhancement in transport is related to non-ideal MHD (tearing) activity. The measurements of the ion heat temperature suggest that the ion heat pulse is relatively weak and slow, consistent with simple power balance thermal diffusivity modeling. A more interesting observation was that ion and electron heat pulses apparently triggered by the partial sawtooth crash in compound sawteeth in the same plasma were found to propagate at a rate consistent with the power balance thermal diffusivity. These results suggest that the strong ballistic effect seen in sawtooth induced electron heat pulses is caused by the sawtooth mechanism rather, than a strong non-linearity in $\chi$. 


\section{Acknowledgements}

One of the authors (E.F.) would like to thank the DIII-D group for the opportunity to have worked at that facility. This work is supported under DoE Contracts DE-AC0276CH03073 and DE-AC03-89ER51114.15. 


\section{Bibliography}

[1] S. Von Goeler, W. Stodiek, and N. Sauthoff, Phys. Rev. Lett. 33, 1201 (1974).

[2] B.B. Kadomtsev, Fiz. Plazmy I, 710 (1975) [Sov. J. Plasma Phys. I, 389 (1975)].

[3] H. Soltwisch, Rev. Sci. Instrum. 59, 1599 (1988).

[4] K.M. McGuire, et al., Phys. Fluids B 2, (1990) 1287.

[5] W. W. Heidbrink, E. D. Fredrickson, T. K. Mau, C. C. Petty, R.I. Pinsker, M. Porkolab, B.W. Rice, Nucl. Fusion 39, 1369 (1999).

[6] Zakharov, L., G. Rogers, S. Migliuolo, Phys. Fluids B 5, 2498 (1993).

[7] F. Levinton, Rev. Sci. Instrum. 63, 5157 (1992).

[8] B. W. Rice, K.H. Burrel, R.J. Groebner, R.P. Seraydarian, Rev. Sci. Instrum. 61 (1999) 815.

[9] R. V. Budny, Nucl. Fusion 34, 1247 (1994), and references therein.

[10] D. Wroblewski and R.T. Snider, Phys. Rev. Lett. 71, 859 (1993).

[11] F. Levinton, L. Zakharov, S. Batha, J. Manickam, M.C. Zarnstorff, Phys. Rev. Lett. 72, 2895 (1994).

[12] A. Cavallo, R.C. Cutler, and M.P. McCarthy, Rev. Sci. Instrum. 59, 889 (1988).

[13] Y. Nagayama, K.M. McGuire, M. Bitter, E.D. Fredrickson, K.W. Hill, H. Hsuan, A. Janos, W. Park, G. Taylor, and M. Yamada, Phys. Rev. Lett. 67, 3527 (1991).

[14] Y. Nagayama, M. Yamada, W. Park, E. Fredrickson, A.C. Janos, K.M. McGuire, and G. Taylor, Phys. Plasmas 3, 1647 (1996)

[15] W. Park, D.A. Monticello, E. Fredrickson, and K.McGuire, Phys. Fluids B 3, 507 (1991).

[16] E. D. Fredrickson, K. McGuire, A. Cavallo, R. Budny, et al., Phys. Rev. Lett. 65, 2869 (1990).

[17] W. Park, E.D. Fredrickson, A. Janos, J. Manickam, and W.M. Tang, Phys. Rev. Lett. 75, 1763 (1995).

[18] K.M. McGuire, V. Arunasalam, M.G. Bell, et al., in Plasma Physics and Controlled Nuclear Fusion Research 1986, Kyoto (International Atomic Energy Agency, Vienna, 1987), Vol. 1, p. 421. 
[19] E.D. Fredrickson, K. McGuire, Z. Chang, A. Janos, M. Bell, R.V. Budny, C. E. Bush, J. Manickam, H. Mynick, R. Nazikian, and G. Taylor, Phys. Plasmas 2, 4216 (1995).

[20] E.D. Fredrickson, K.M. McGuire, Z.Y. Chang, A. Janos, J. Manickam, G. Taylor, S. Mirnov, I. Semenov, D. Kislov, and D. Martynov, Phys. Plasmas 3, 2620 (1996).

[21] Y. Nagayama and A.W. Edwards, Rev. Sci. Instrum. 63, 4757 (1992).

[22] J.D. Callen and G.L. Jahns, Phys. Rev. Lett. 38, 971 (1977)

[23] M. Soler and J.D. Callen, Nucl. Fusion 19, 703 (1979)

[24] E.D. Fredrickson, J.D. Callen, K.M. McGuire, J.D. Bell, R.J. Colchin, P.C. Efthimion, K.W. Hill, R. Izzo, D.R. Mikkelsen, D.A. Monticello, V. Paré, G. Taylor, and M. Zarnstorff, Nucl. Fusion 26, 849 (1986).

[25] E.D. Fredrickson, A.C. Janos, K.M. McGuire, S.D. Scott, G. Taylor and Z. Chang, Nucl. Fusion 33, 1759 (1993).

[26] H. T. Evensen, R. J. Fonck, S. F. Paul, S. D. Scott, Nucl. Fusion 39, 133 (1999).

[27] W. Pfeiffer, Nucl. Fusion 25, 673 (1985).

[28] R. Kaita, R.B. White, A.W. Morris, E.D. Fredrickson, K.M. McGuire, S.S. Medley, T.J. Murphy, and S.D. Scott, Phys. Fluids B 2, 1584 (1990).

[29] E.D. Fredrickson, K.M. McGuire, Y. Nagayama, M.G. Bell, A. Cavallo, P.C. Efthimion, H. Fleischmann, A.C. Janos, D.W. Johnson, D.K. Mansfield, D.A. Monticello, H.K. Park, W. Park, W. Stodiek, G. Taylor, M. Ulrickson et.al., Plasma Phys. and Controlled Nuclear Fusion Research, Washington 1990 (Intern. Atomic Energy Agency, Vienna, 1991), Vol. 1, 559.

[30] R. Nazikian, N. Bretz, E. Fredrickson, Y. Nagayama, E. Mazzucato, K. McGuire, H.K. Park, G. Taylor, A. Cavallo, M. Diesso and J. Felt, in Proceedings of the18th European Physical Society Conf. on Controlled Fusion and Plasma Physics 1991, Berlin (The European Physical Society, Geneva, 1996), Vol. 15c, Part I, p. 265. 


\section{Figure Captions}

1a. Plasma Current and Neutral Beam Injection power and Ion Cyclotron Range of Frequency heating power for a typical shot in this experiment on DIII-D. The central electron temperature trace is shown to indicate sawtooth activity.

1b. Plasma Current and Neutral Beam Injection power and Ion Cyclotron Range of Frequency heating power for a comparison shot in on TFTR. The central electron temperature trace is shown to indicate sawtooth activity.

2a. Radial profiles of the safety factor $q$ for DIII-D deduced using EFIT and MSE data. The profile with $\mathrm{q}(0)<1$ is approximately $10 \mathrm{msec}$ before the sawtooth crash, the other profile is approximately $10 \mathrm{msec}$ after the crash.

2b. Radial profile of the safety factor for the TFTR plasma as calculated with TRANSP.

3. Contour plot of the electron temperature vs. major radius and time showing the $\mathrm{m}=1, \mathrm{n}=1$ sawtooth precursor on TFTR. The growth of the $(1,1)$ island starts at the time indicated by the arrow. $\left(\beta_{\mathrm{pol}}=0.44, \beta_{\mathrm{n}}=0.9, \mathrm{I}_{\mathrm{P}}=2.47 \mathrm{MA}, \mathrm{q}(\mathrm{a})=3.9, \mathrm{~B}_{\text {tor }}=5.1\right.$ $\mathrm{T}, \mathrm{P}_{\mathrm{NBI}}=19 \mathrm{MW},\left\langle\mathrm{n}_{\mathrm{e}}>=3.3 \times 10^{19} \mathrm{~m}^{-3}\right.$ )

4. Poloidal magnetic field fluctuation amplitude shown vs. the width of the $(m, n)=(1,1)$ sawtooth precursor island for the sawtooth in Fig. 3.

5. Contour plot of the electron temperature vs. major radius and time showing the $m=1, n=1$ sawtooth precursor on TFTR shot 76642 . The growth of the $(1,1)$ island starts at the time indicated by the arrow. $\left(\beta_{\mathrm{pol}}=0.4, \beta_{\mathrm{n}}=0.8, \mathrm{I}_{\mathrm{P}}=2.47 \mathrm{MA}, \mathrm{q}(\mathrm{a})=\right.$ $3.9, \mathrm{~B}_{\text {tor }}=5.1 \mathrm{~T}, \mathrm{P}_{\mathrm{NBI}}=19 \mathrm{MW},\left\langle\mathrm{n}_{\mathrm{e}}>=3.3 \times 10^{19} \mathrm{~m}^{-3}\right.$ ) 
6. Contour plot of the electron temperature vs. major radius and time showing the final stage of the $m=1, n=1$ sawtooth precursor on TFTR shot 76649. A moderate $n$ ballooning mode can be seen in the last period. $\left(\beta_{\mathrm{pol}}=0.4, \beta_{\mathrm{n}}=0.8, \mathrm{I}_{\mathrm{P}}=2.47 \mathrm{MA}\right.$, $\mathrm{q}(\mathrm{a})=3.9, \mathrm{~B}_{\text {tor }}=5.1 \mathrm{~T}, \mathrm{P}_{\mathrm{NBI}}=19 \mathrm{MW},\left\langle\mathrm{n}_{\mathrm{e}}>=3.3 \times 10^{19} \mathrm{~m}^{-3}\right)$

7. A contour plot of the electron temperature vs. major radius and time for a sawtooth crash on DIII-D. The behavior is similar to that seen for the sawtooth on TFTR in Figure 3. Of particular interest is that the heat pulse can be seen to start at roughly the same time as the growth of the $(1,1)$ island. $\left(\beta_{\mathrm{pol}}=0.46, \beta_{\mathrm{n}}=1.17, \mathrm{I}_{\mathrm{P}}=1.2 \mathrm{MA}, \kappa\right.$ $=1.44, \mathrm{q}_{95}=4.0, \mathrm{~B}_{\text {tor }}=1.86 \mathrm{~T}, \mathrm{P}_{\mathrm{NBI}}=5 \mathrm{MW},\left\langle\mathrm{n}_{\mathrm{e}}>=3 \times 10^{19} \mathrm{~m}^{-3}\right.$ )

8a. A growing $m=1, n=1$ island precursor to a sawtooth crash on DIII-D. From the shape of the electron temperature contours it is possible to deduce that the island is crescent shaped in the poloidal cross-section and that the core is (nominally) round. $\left(\beta_{\mathrm{pol}}=0.46, \beta_{\mathrm{n}}=1.23, \mathrm{I}_{\mathrm{P}}=1.2 \mathrm{MA}, \kappa=1.44, \mathrm{q}_{95}=3.7, \mathrm{~B}_{\text {tor }}=1.77 \mathrm{~T}, \mathrm{P}_{\mathrm{NBI}}=5 \mathrm{MW}\right.$, $<\mathrm{n}_{\mathrm{e}}>=3 \times 10^{19} \mathrm{~m}^{-3}$ )

8b. A reconstruction of the data shown in Fig. 7a into a 2-D image in the poloidal crosssection.

9a. The redistribution of heat as expected from the Kadomtsev model of the sawtooth reconnection using the q-profile as measured with the MSE system on DIII-D and the pre-crash electron temperature profile from the sawtooth shown in Fig. 7. The predicted post-crash profile is compared to the measured post-crash temperature profile.

9b. Simulation of the time dependent electron temperature profile through a sawtoothlike event. The pre-crash temperature profile and two post crash profiles are shown. The first post-crash profile is immediately after the simulated reconnection and the second is $200 \mu \mathrm{sec}$ after the reconnection. 
10. Simulations of DIII-D electron temperature heat pulses using the power balance thermal diffusivity and a simple sawtooth model (dashed line) compared to measured heat pulses (solid line).

11. Simulations of DIII-D electron temperature heat pulses using the measured temperature dependence at $\mathrm{r}=34 \mathrm{~cm}$ as the inner boundary condition and $\chi_{\mathrm{e}} \approx \chi_{\mathrm{e}}{ }^{\mathrm{PB}}$ (dashed line) compared to measured heat pulses (solid line).

12. Simulations of DIII-D electron temperature heat pulses using the measured temperature dependence at $\mathrm{r}=34 \mathrm{~cm}$ as the inner boundary condition and $\chi_{\mathrm{e}} \approx 20$ $\chi_{\mathrm{e}}^{\mathrm{PB}}$ (dashed line) compared to measured heat pulses (solid line).

13. Simulations of TFTR electron temperature heat pulses using the measured temperature dependence at $\mathrm{r}=34 \mathrm{~cm}$ as the inner boundary condition and $\chi_{\mathrm{e}} \approx 20$ $\chi_{\mathrm{e}}{ }^{\mathrm{PB}}$ (dashed line) compared to measured heat pulses (solid line). Same shot as Fig. 6.

14. Contour plot of electron temperature vs. radius and time showing the sawtooth precursor behavior. The precursor appears to originally be a kink, with an island of at most $1 \mathrm{~cm}$ width. The $(1,1)$ island begins to grow between $4.7295 \mathrm{~s}$ and $4.7298 \mathrm{~s}$, but stops, then grows again beginning at $4.7301 \mathrm{~s}$, eventually ending with a full reconnection. Some increase in temperature is detectable out to $r=0.33 \mathrm{~m}$ following the first island growth period.

15. Comparison of measured $\mathrm{T}_{\mathrm{i}}$ sawtooth induced heat pulses to simulated heat pulses using $\chi_{\mathrm{i}} \approx \chi_{\mathrm{i}}^{\mathrm{PB}}$. Same sawtooth as Fig. 7. 
16. Comparison of partial sawtooth induced ion heat pulses measured on DIII-D with the CER system (solid line) with simulated heat pulses using the calculated ion thermal diffusivity from TRANSP (dashed line). Same shot as Fig. 7.

17. Comparison of partial sawtooth induced electron heat pulses (solid line) to a simulation using twice the calculated electron thermal diffusivity from TRANSP (dashed line). Same shot as Fig. 6.

18. Comparison of partial sawtooth induced electron heat pulses (solid line) to a simulation using twice the calculated electron thermal diffusivity from TRANSP (dashed line). Same shot as Fig. 5.

19. Comparison of fishbone burst induced electron heat pulses (solid line) to a simulation using the calculated ion thermal diffusivity from TRANSP (dashed line). Same shot as Fig. 6. 
Figure 1
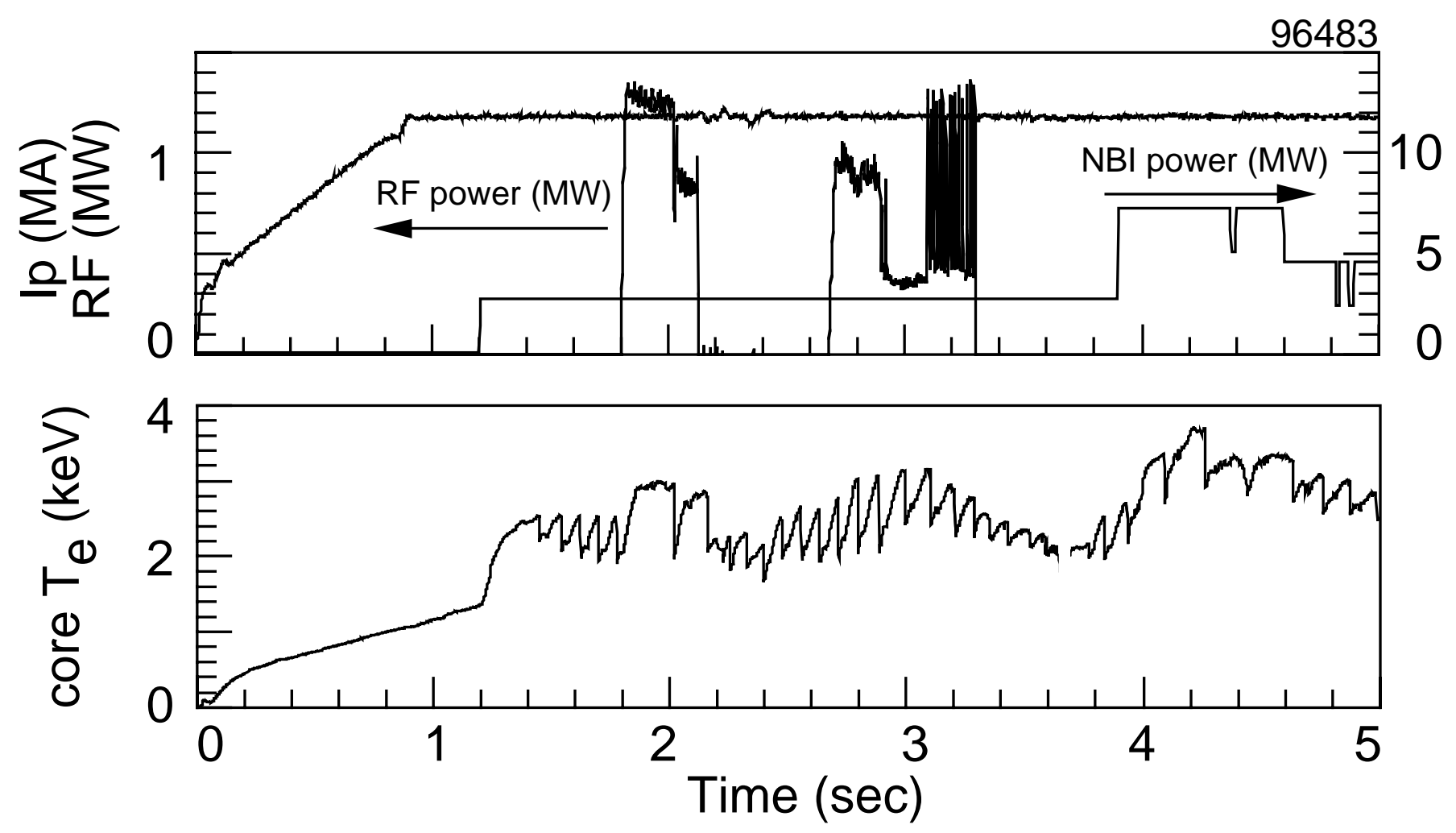

76649
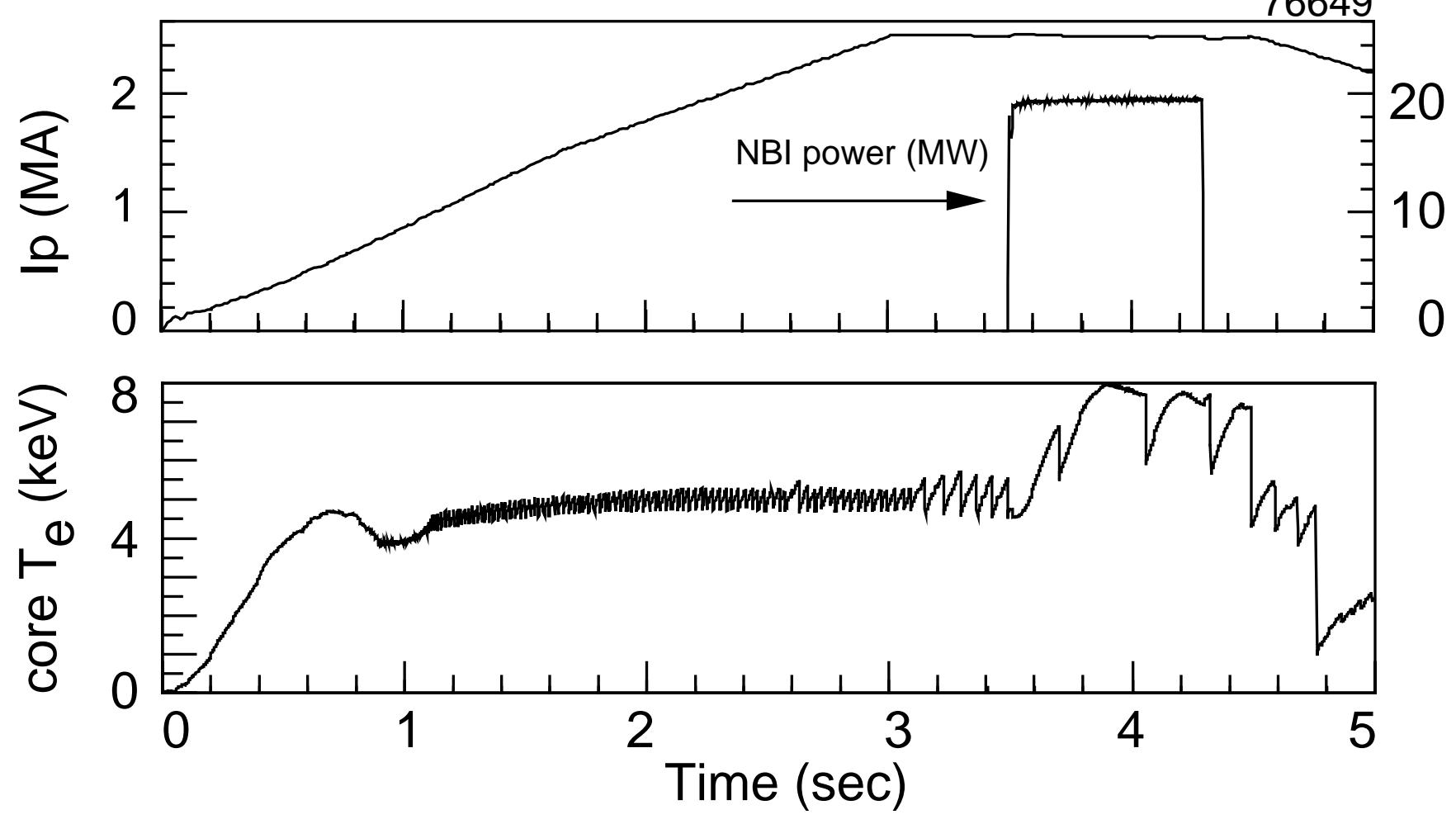
Figure 2
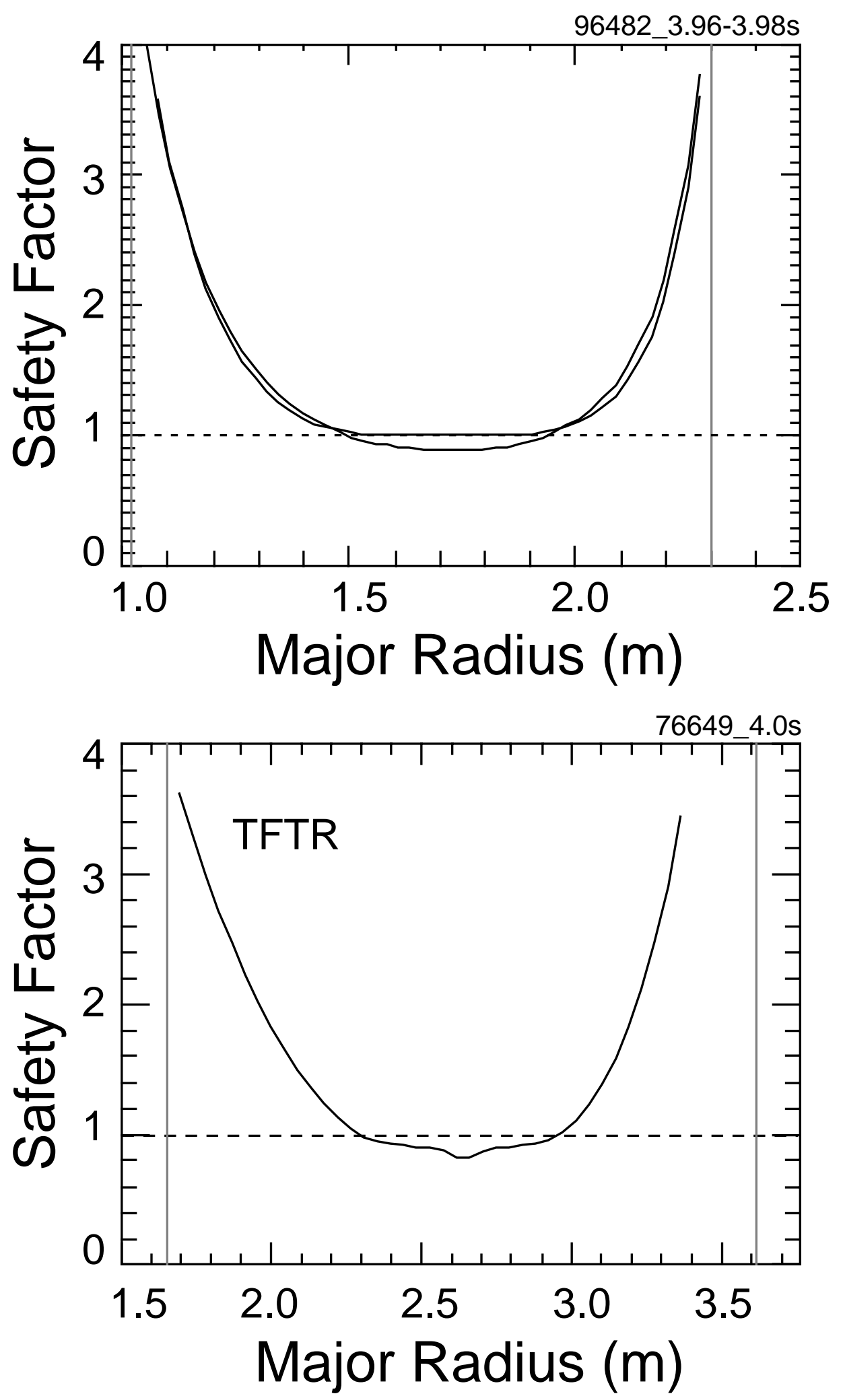
Figure 3

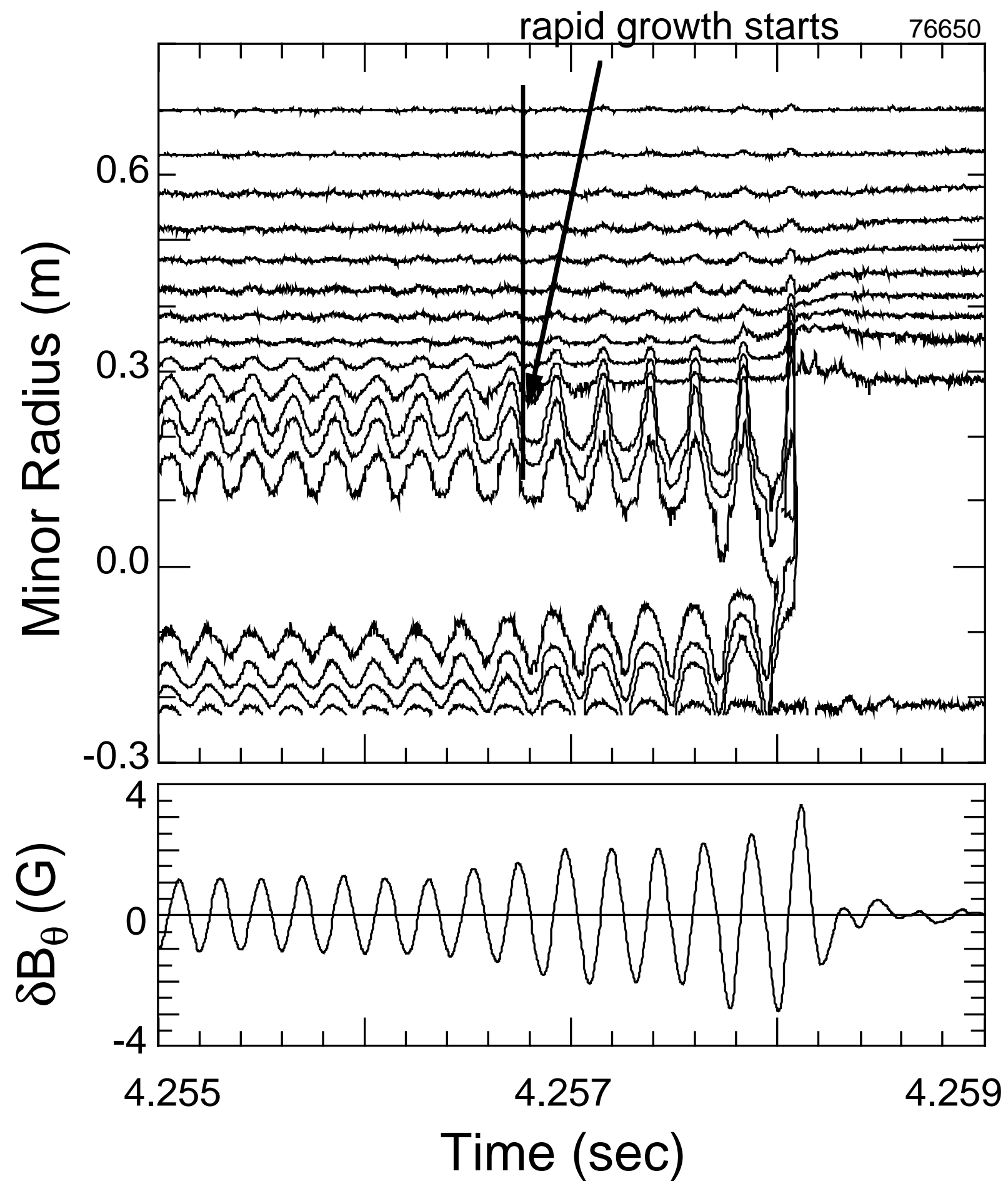


Figure 4

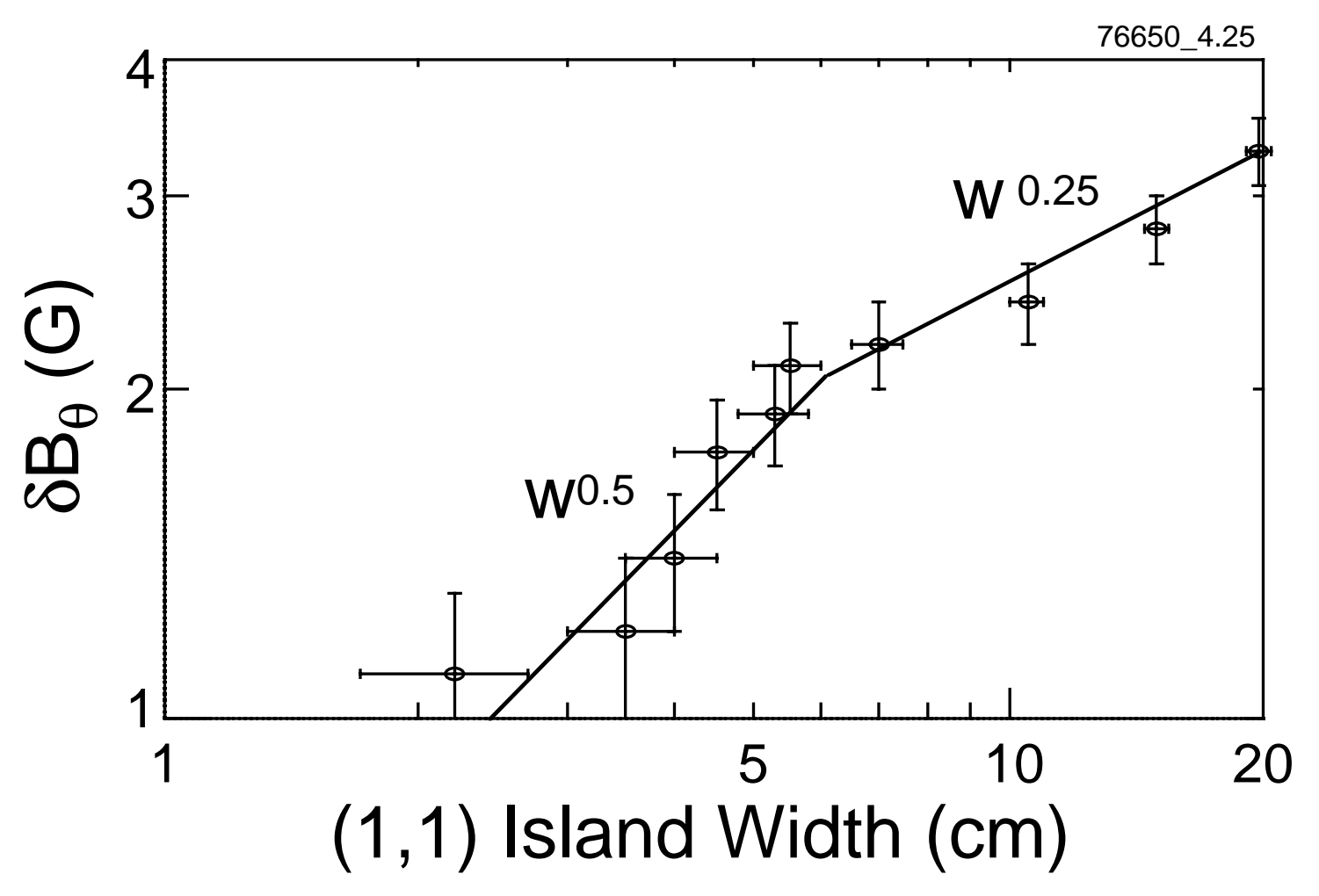


Figure 5

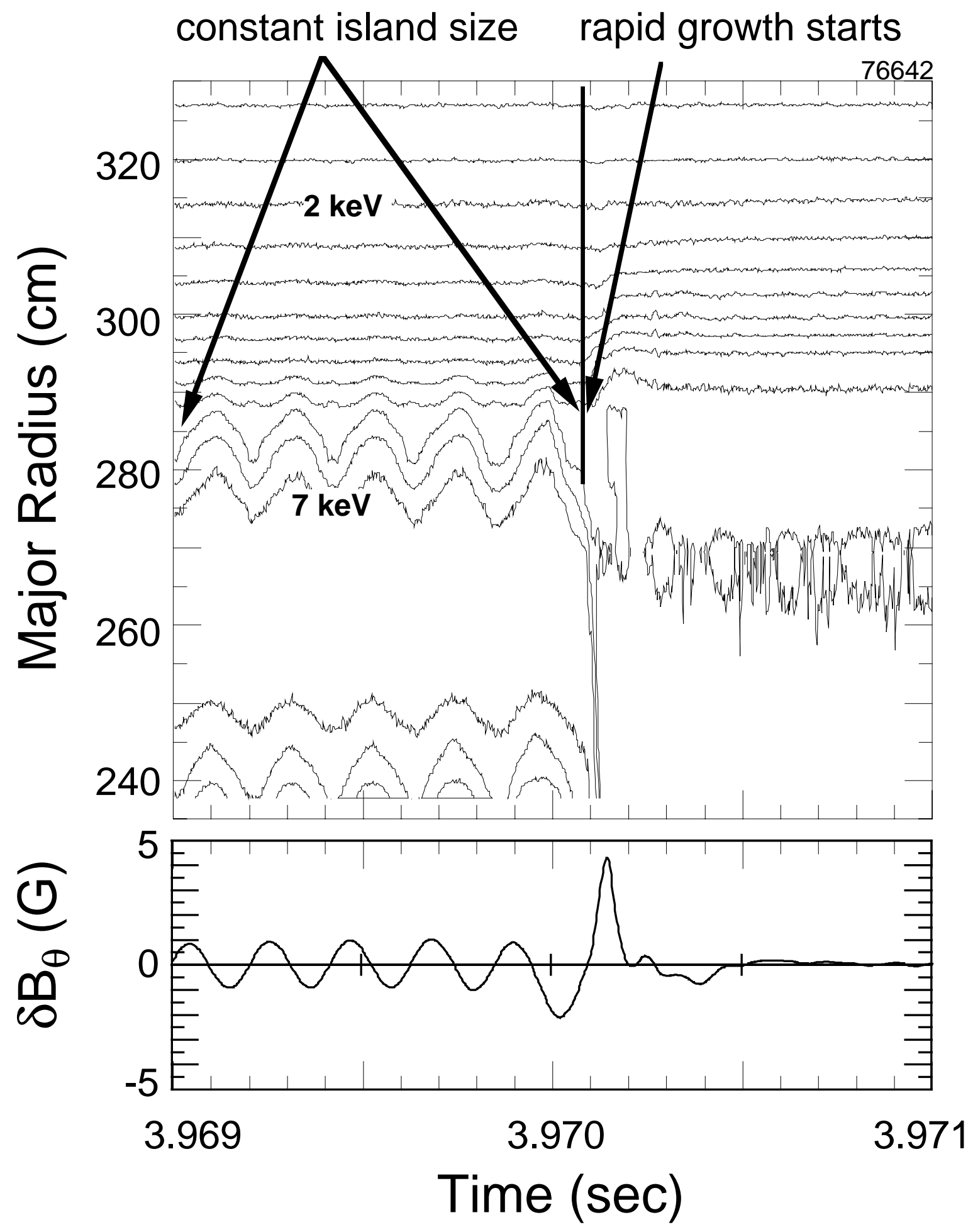




\section{Figure 6}
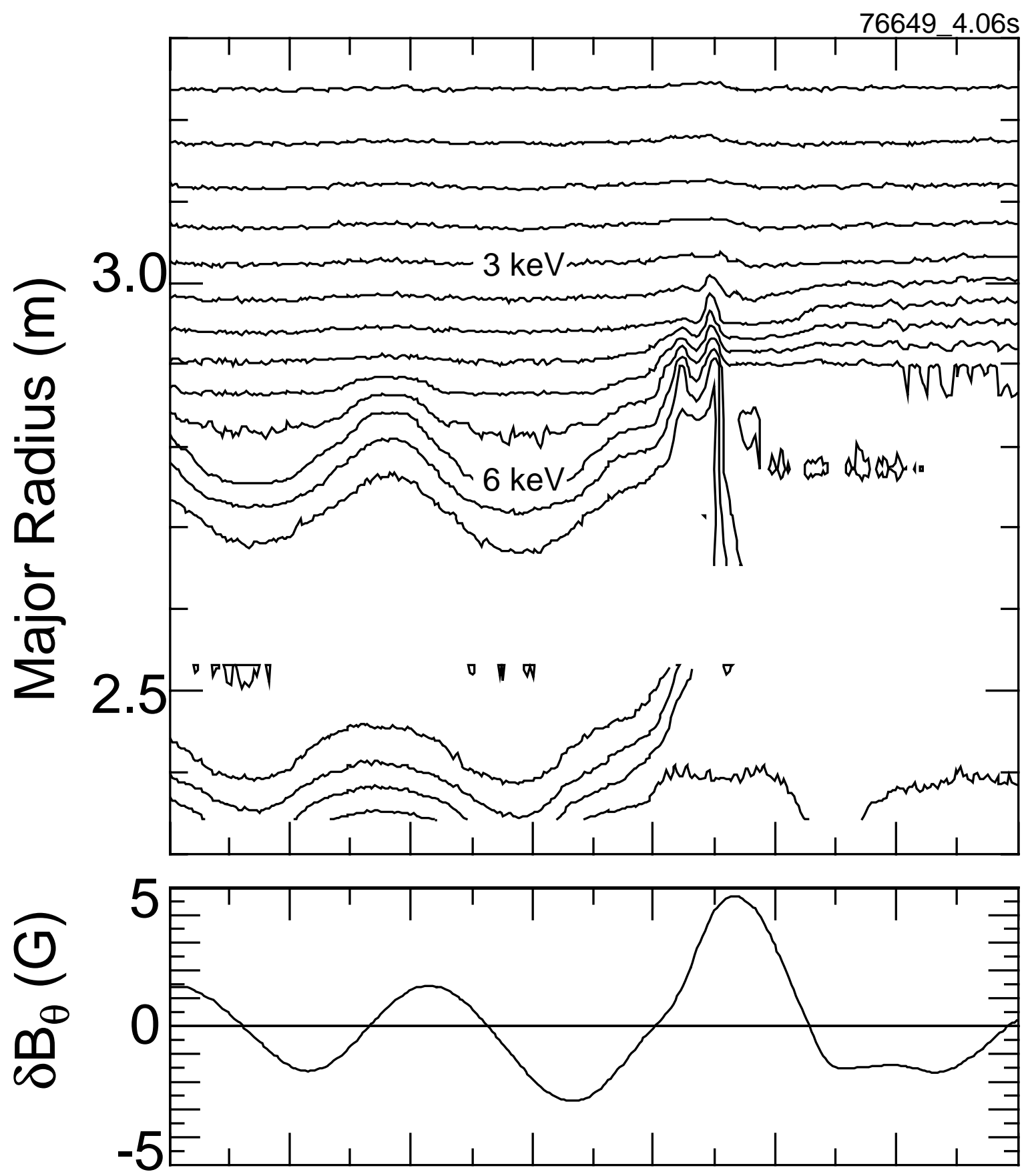
Figure 7

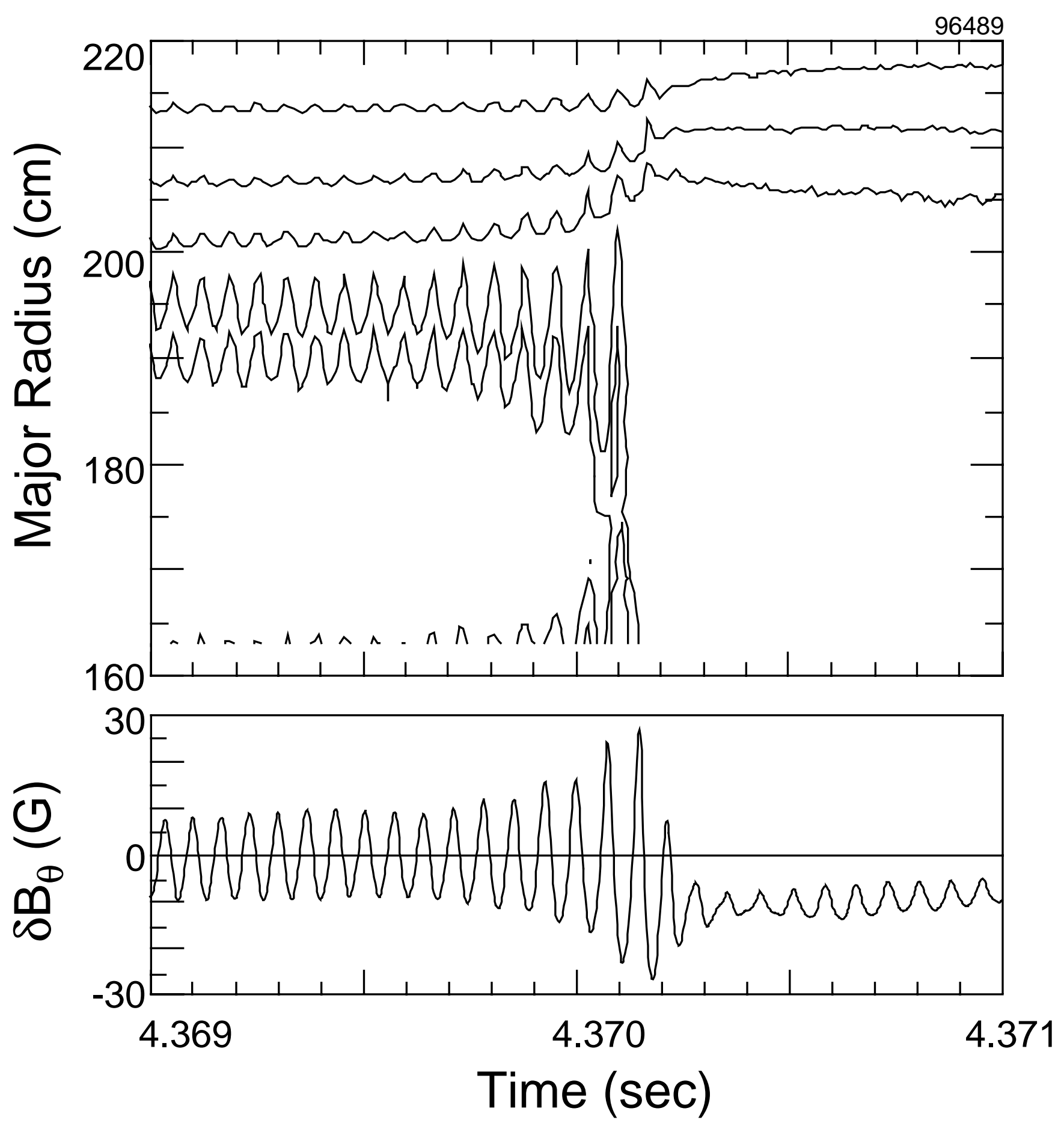


Figure 8a

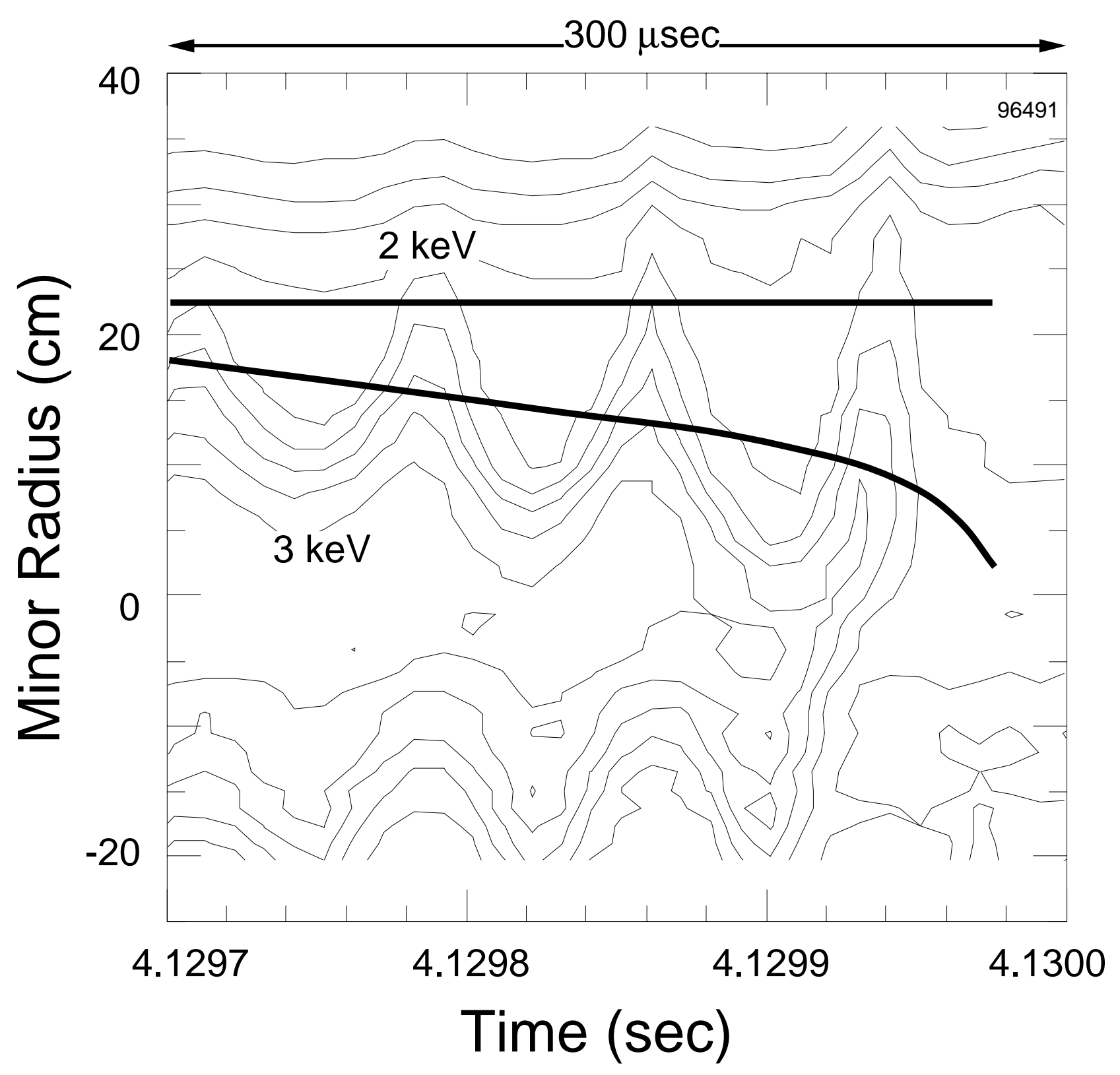


Figure 8b

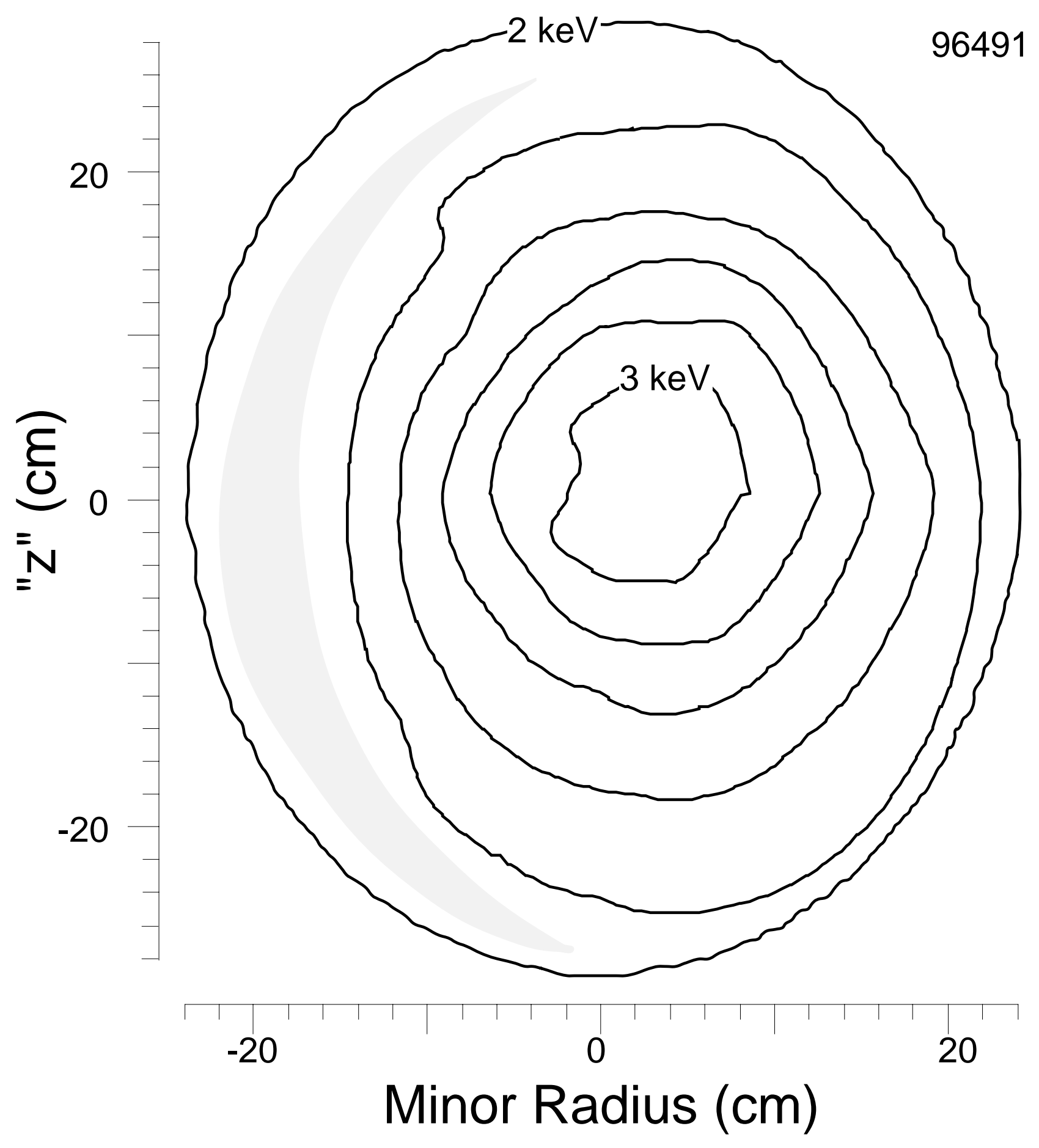


Figure 9
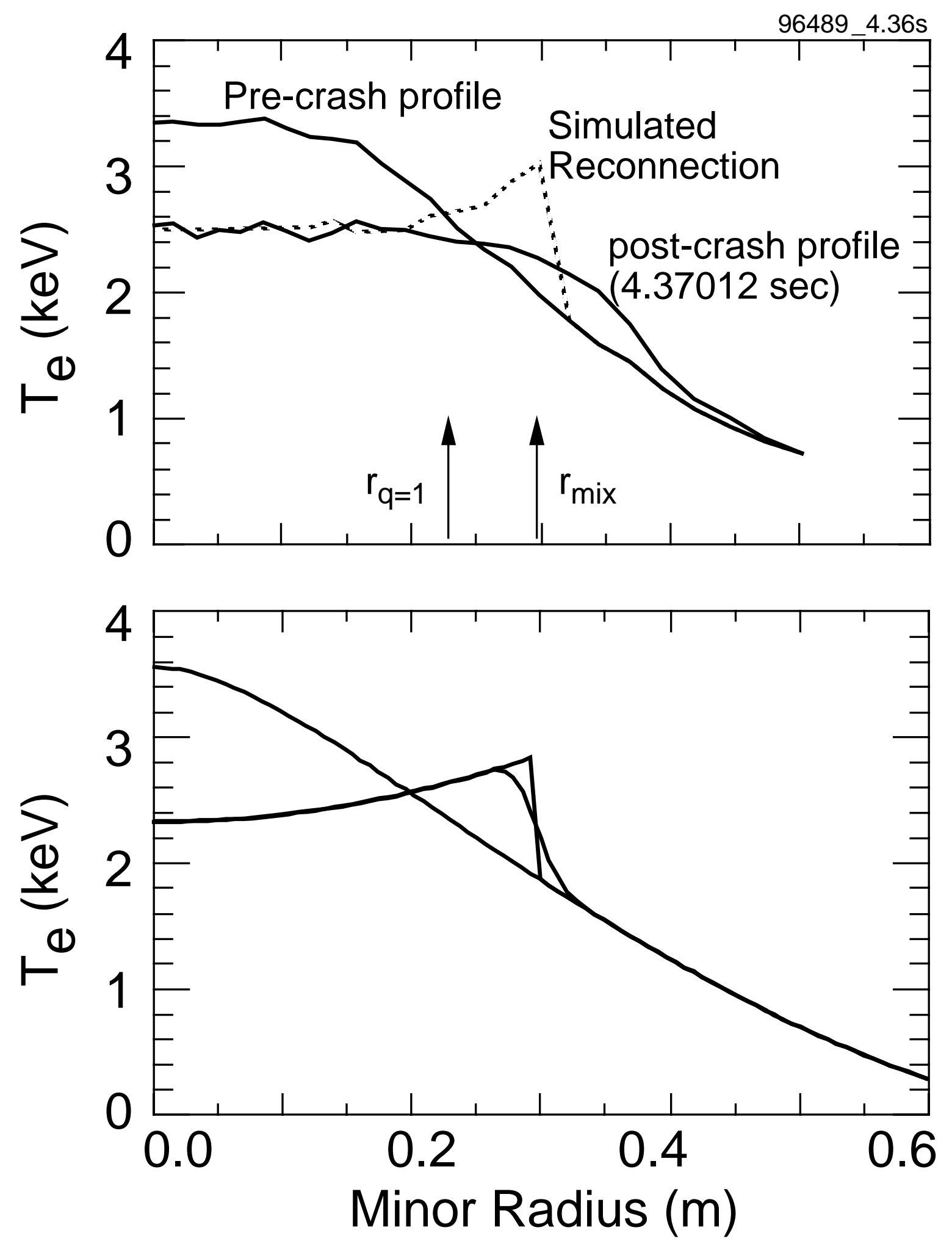
Figure 10

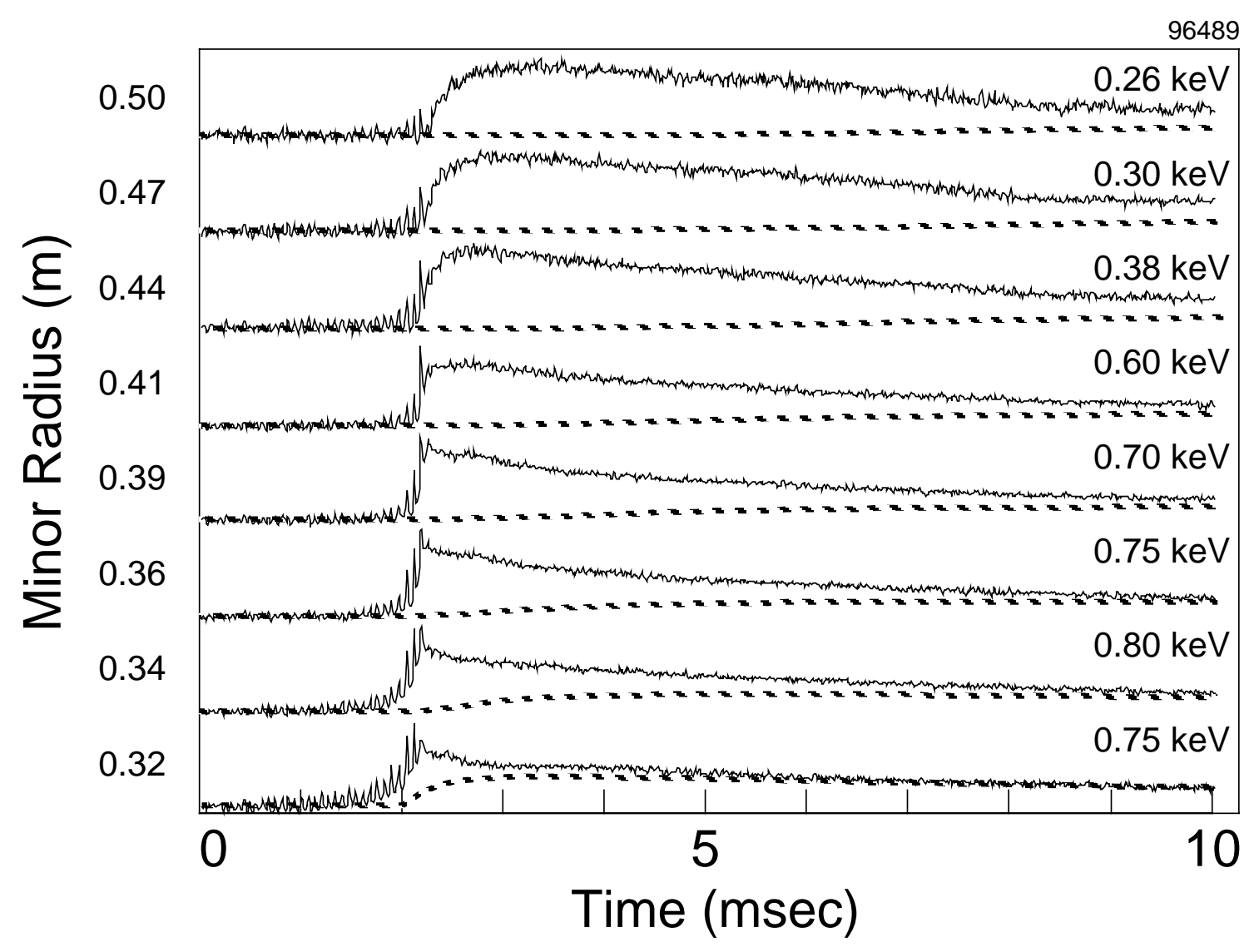


Figure 11

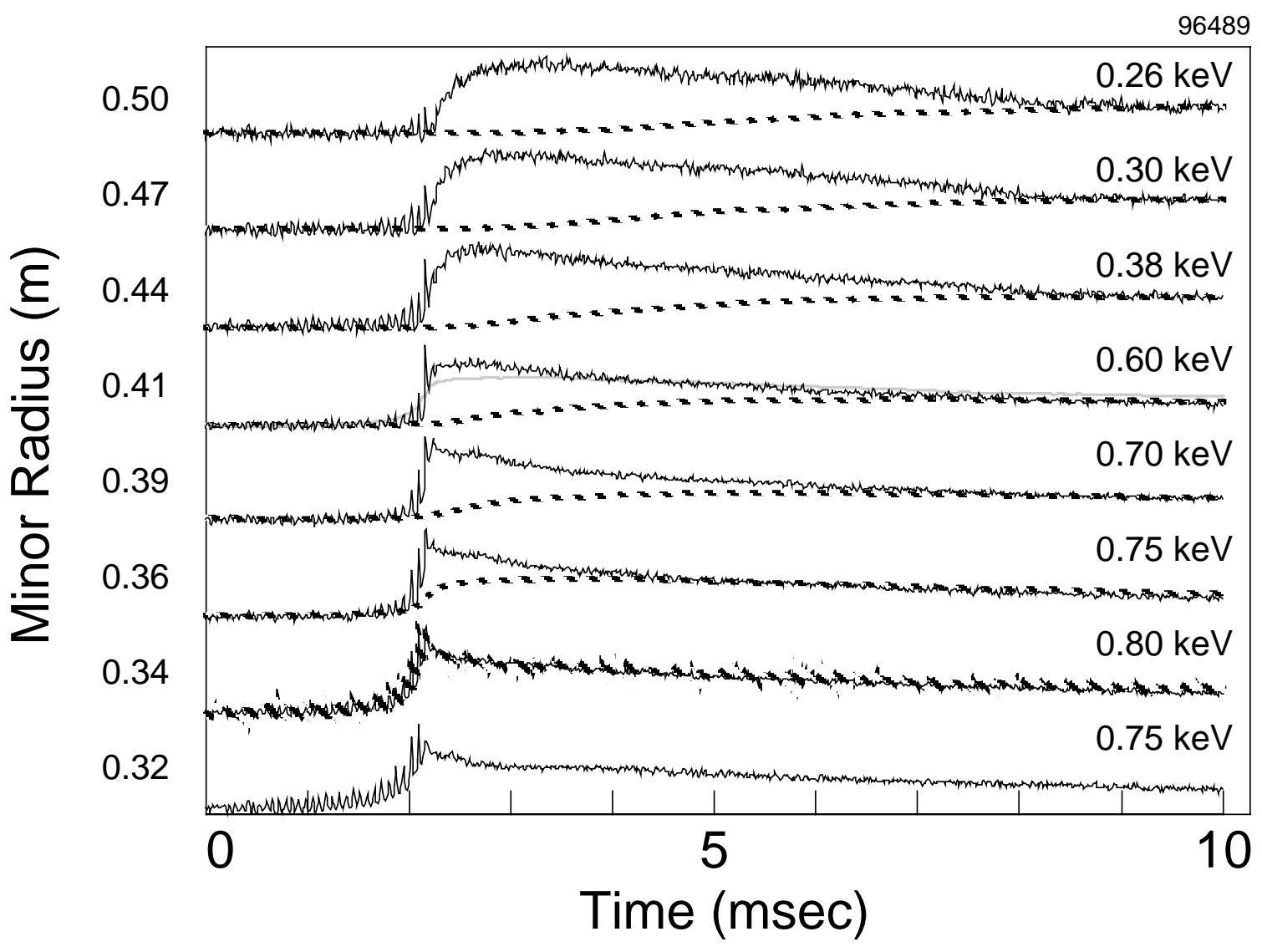


Figure 12

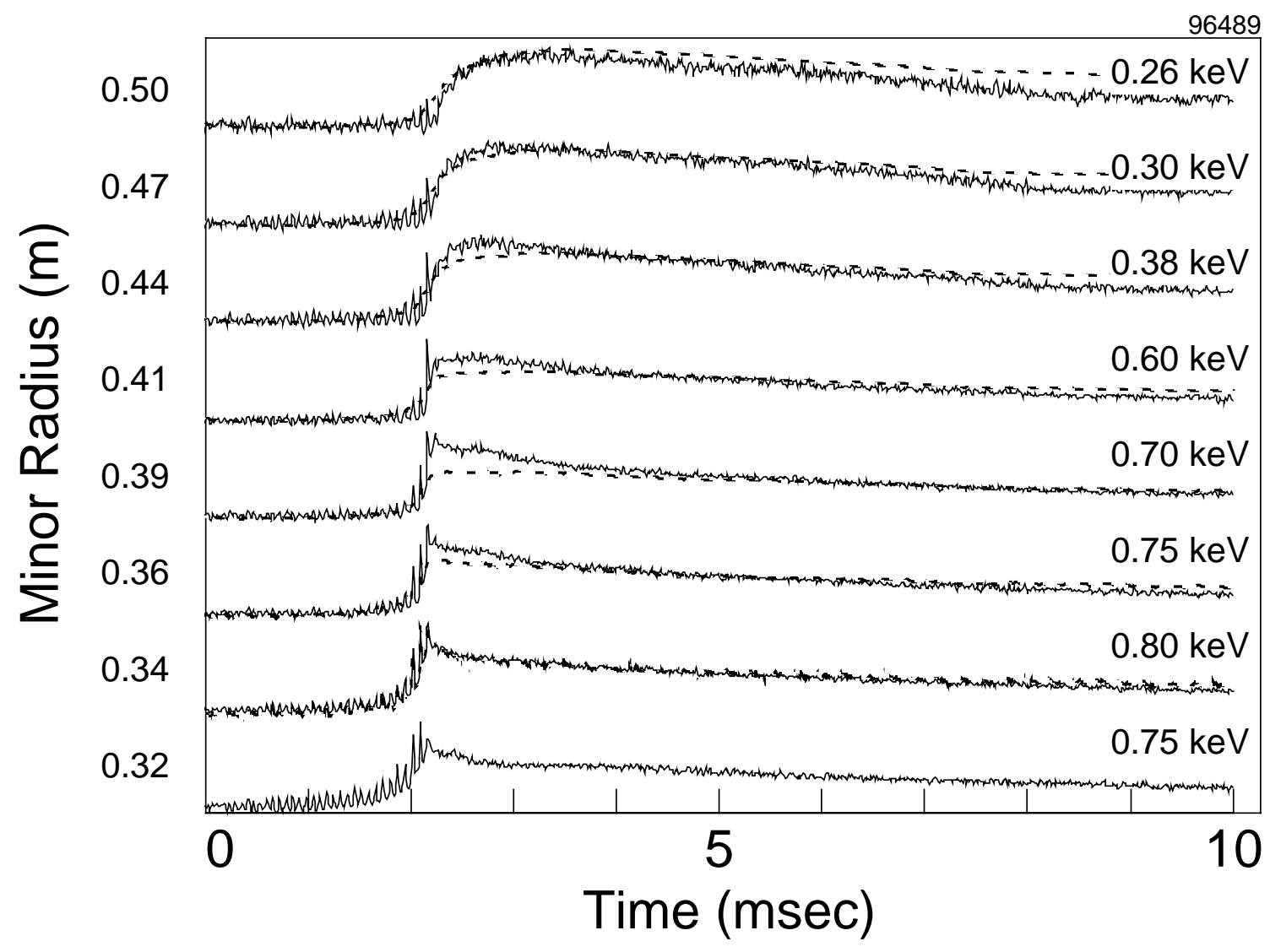


Figure 13

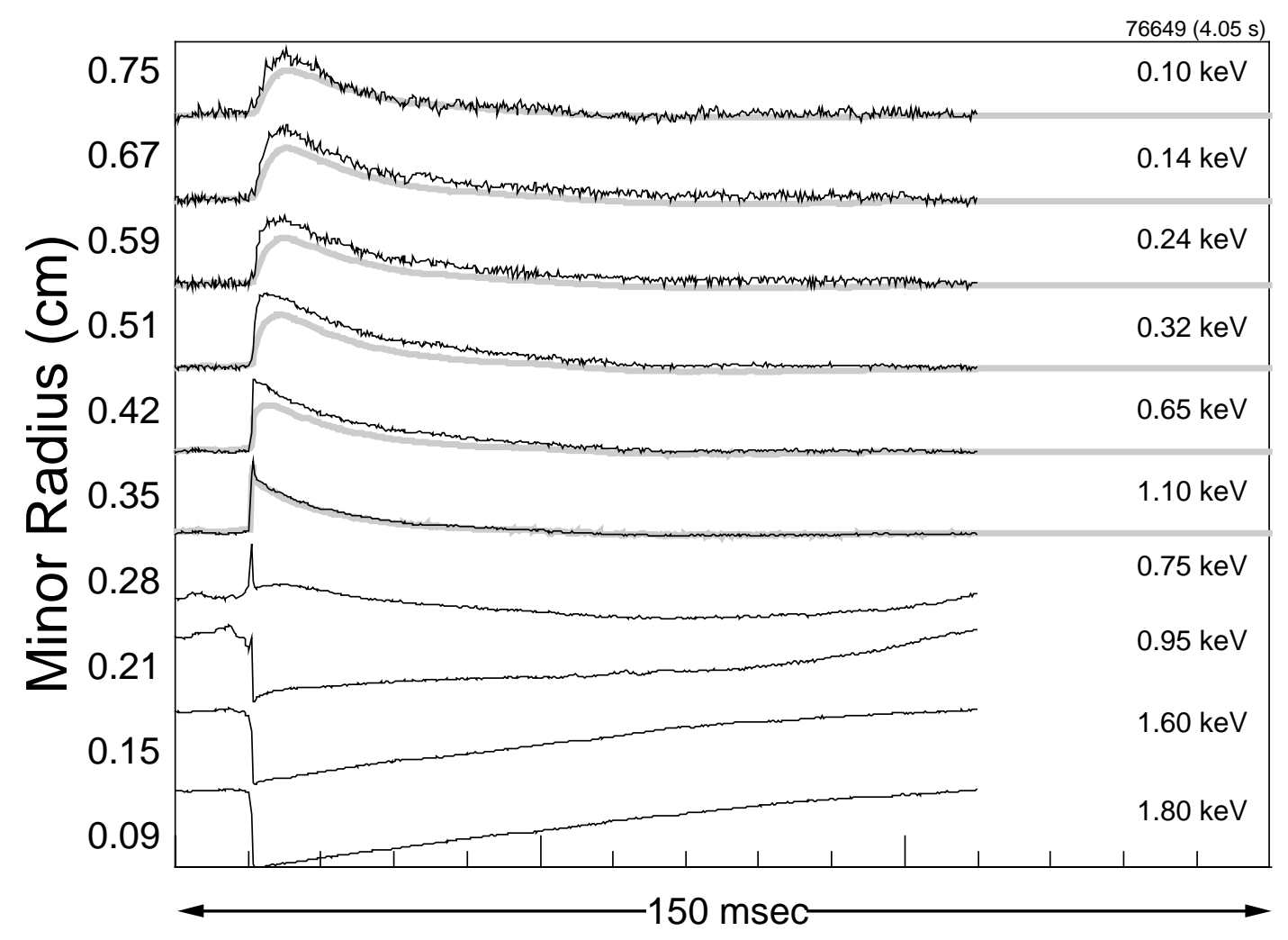


Figure 14

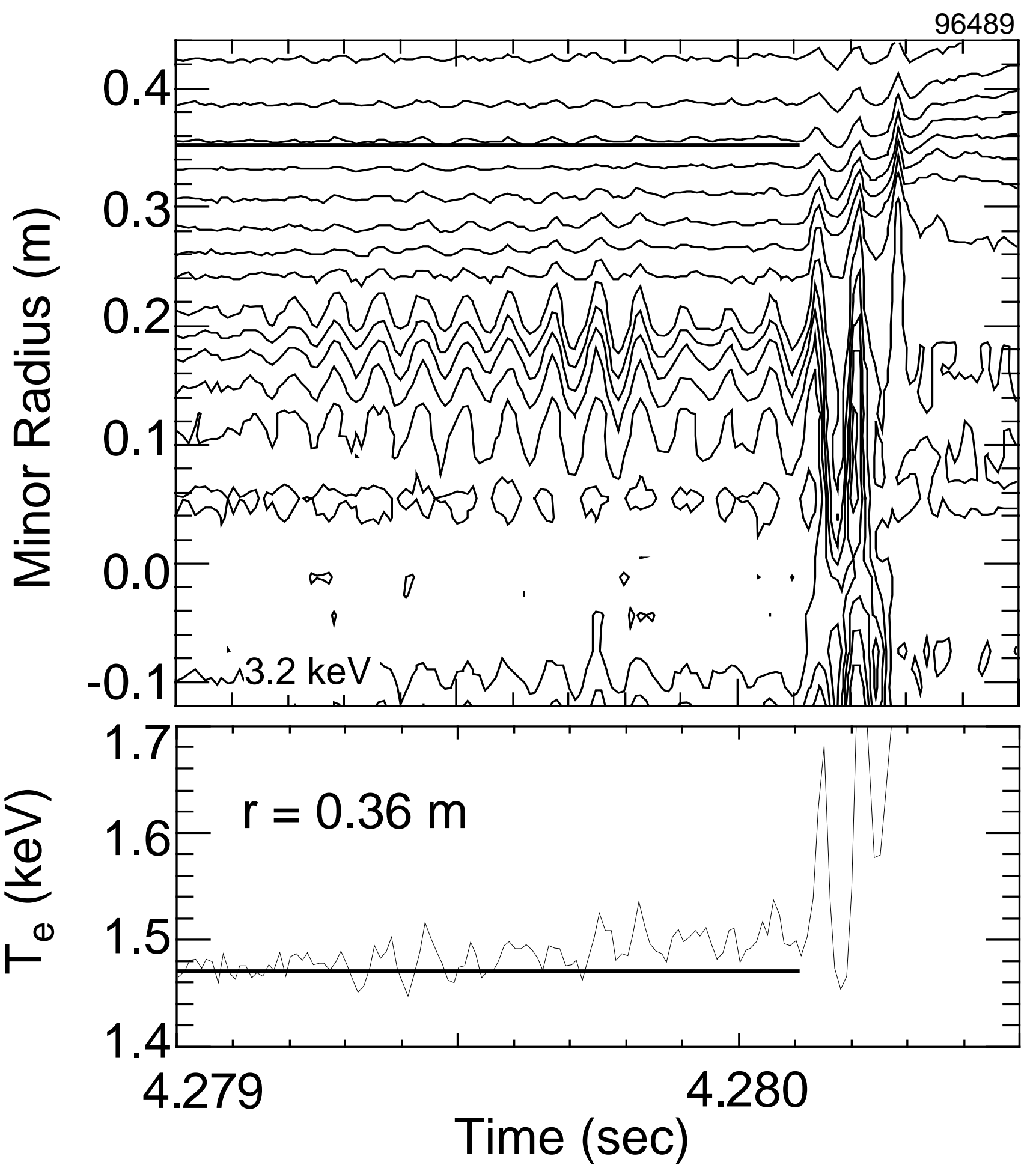


Figure 15

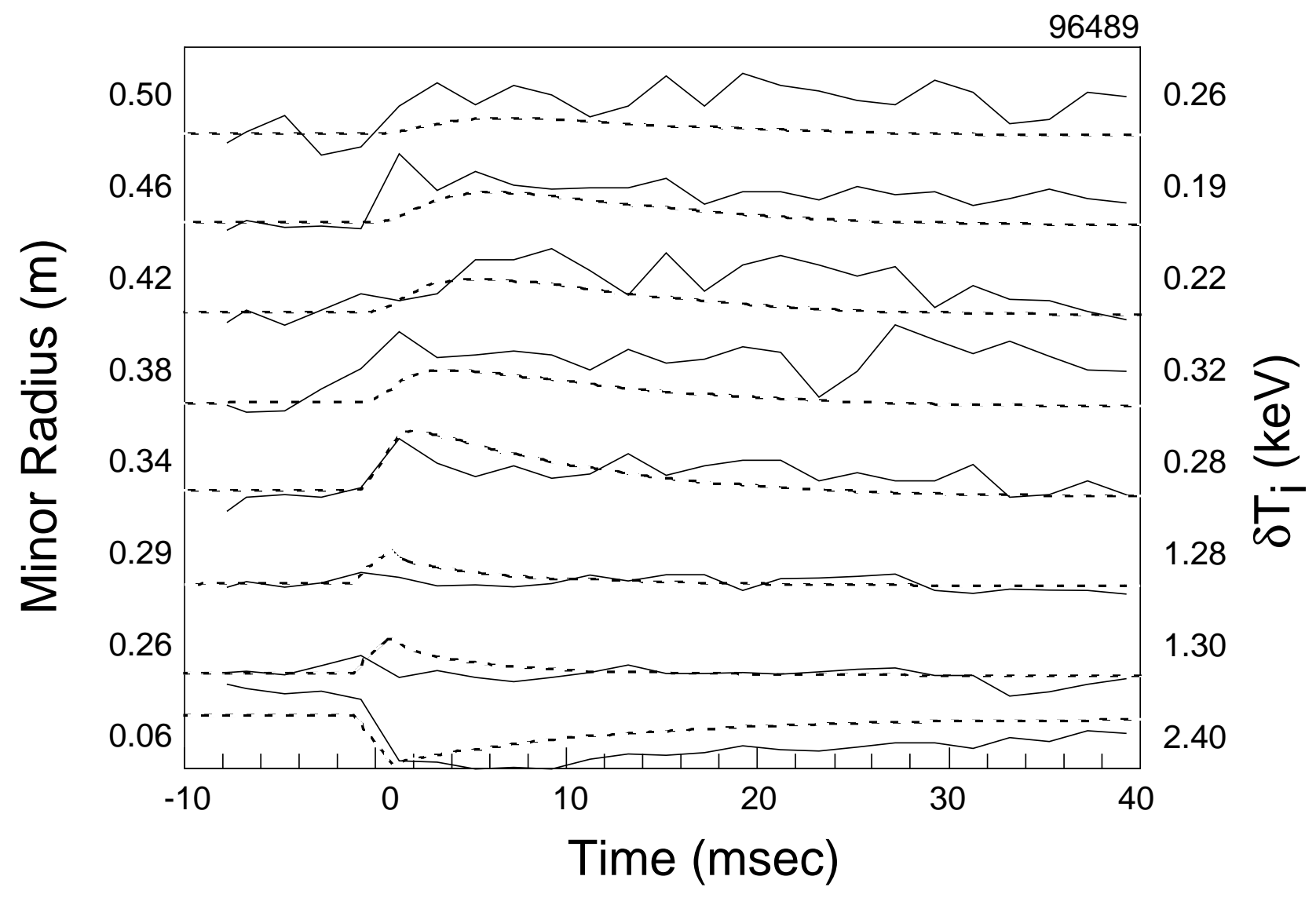


Figure 16

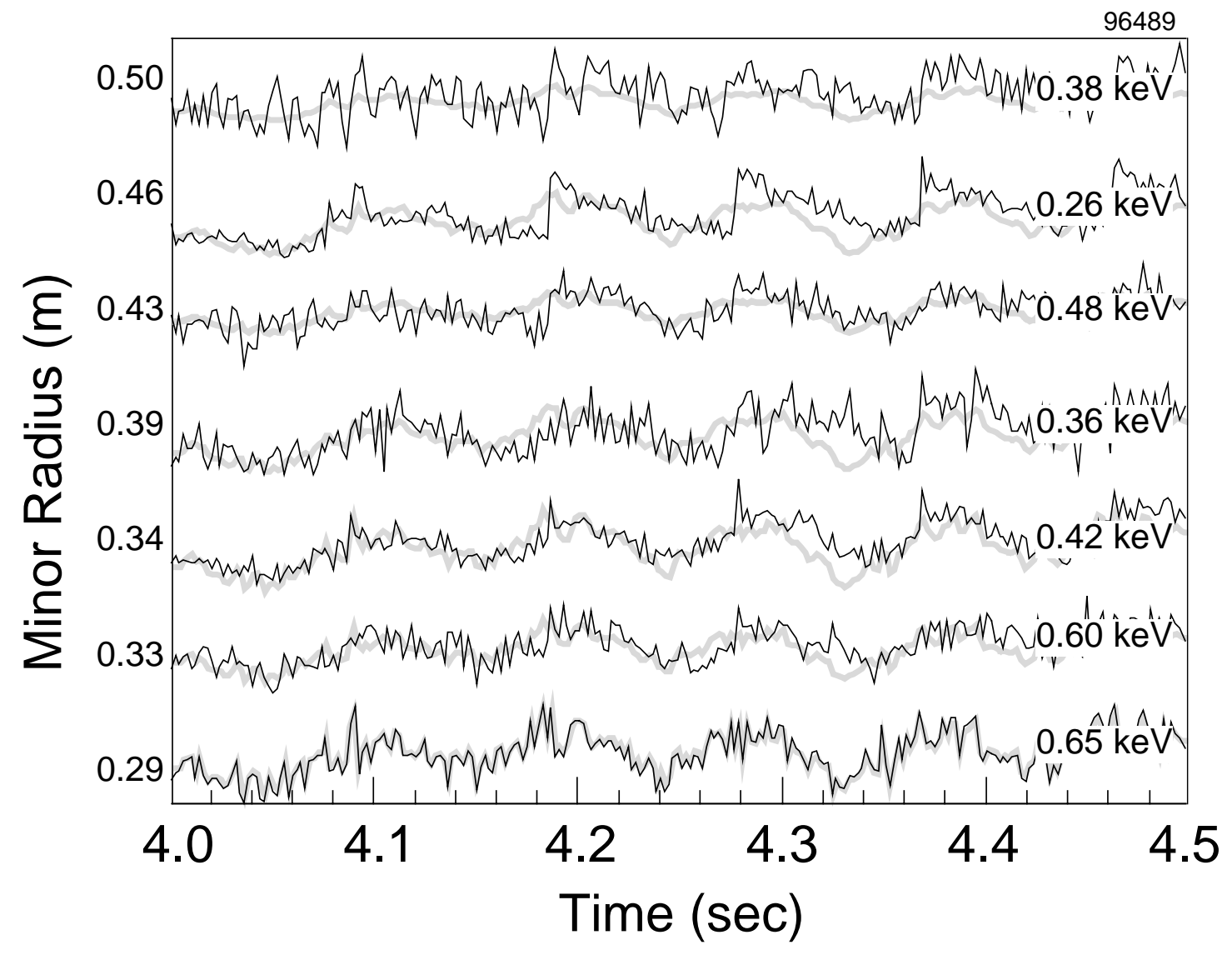




\section{Figure 17}

$$
\chi_{e} \approx 2 \chi_{e}{ }^{P B}=2\left(0.07+3.7 r^{2}\right) /\left(0.3+0.7 \exp \left(-4 r^{2}\right)\right) \mathrm{m}^{2} / \mathrm{s}
$$
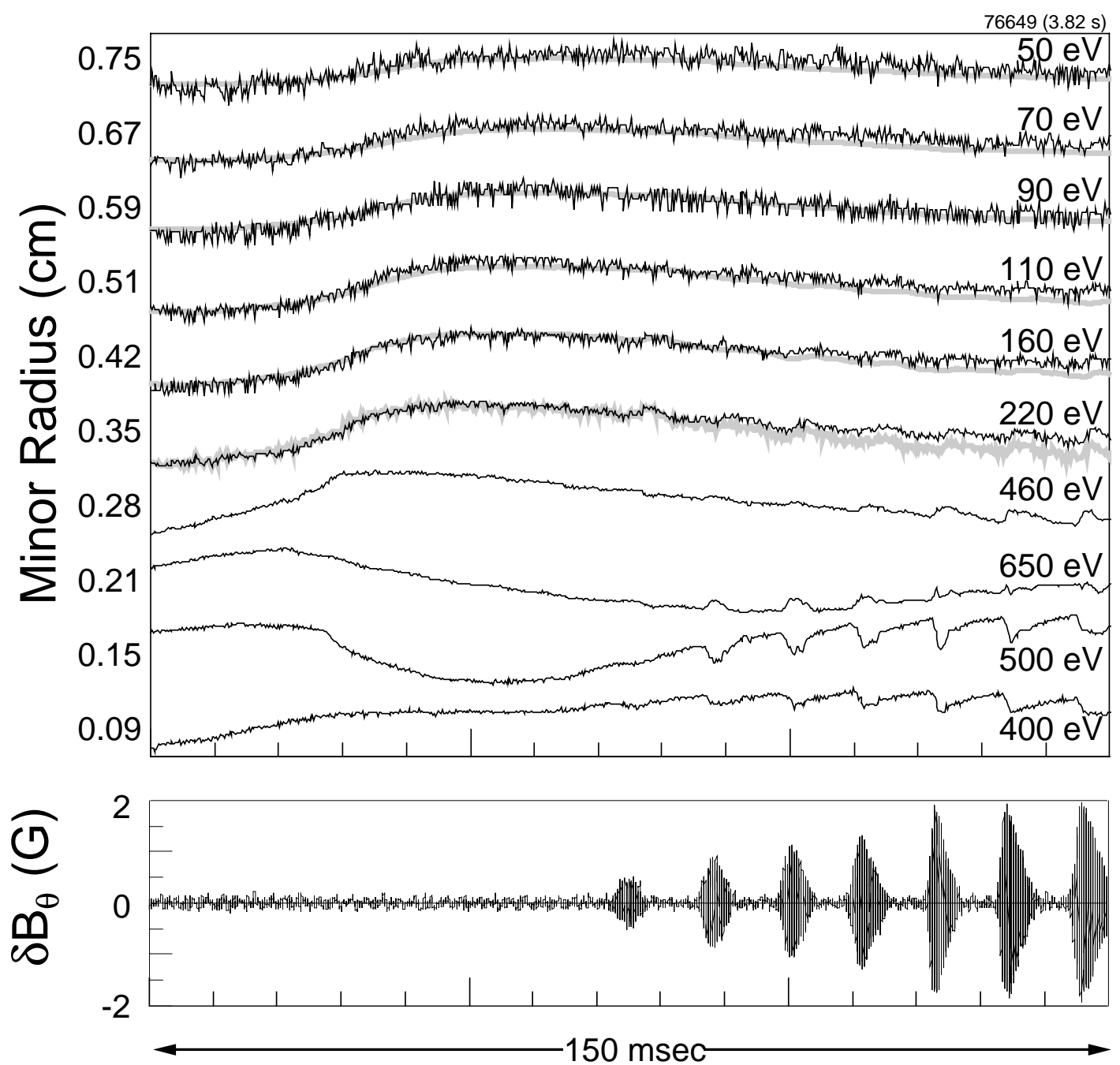


\section{Figure 18}
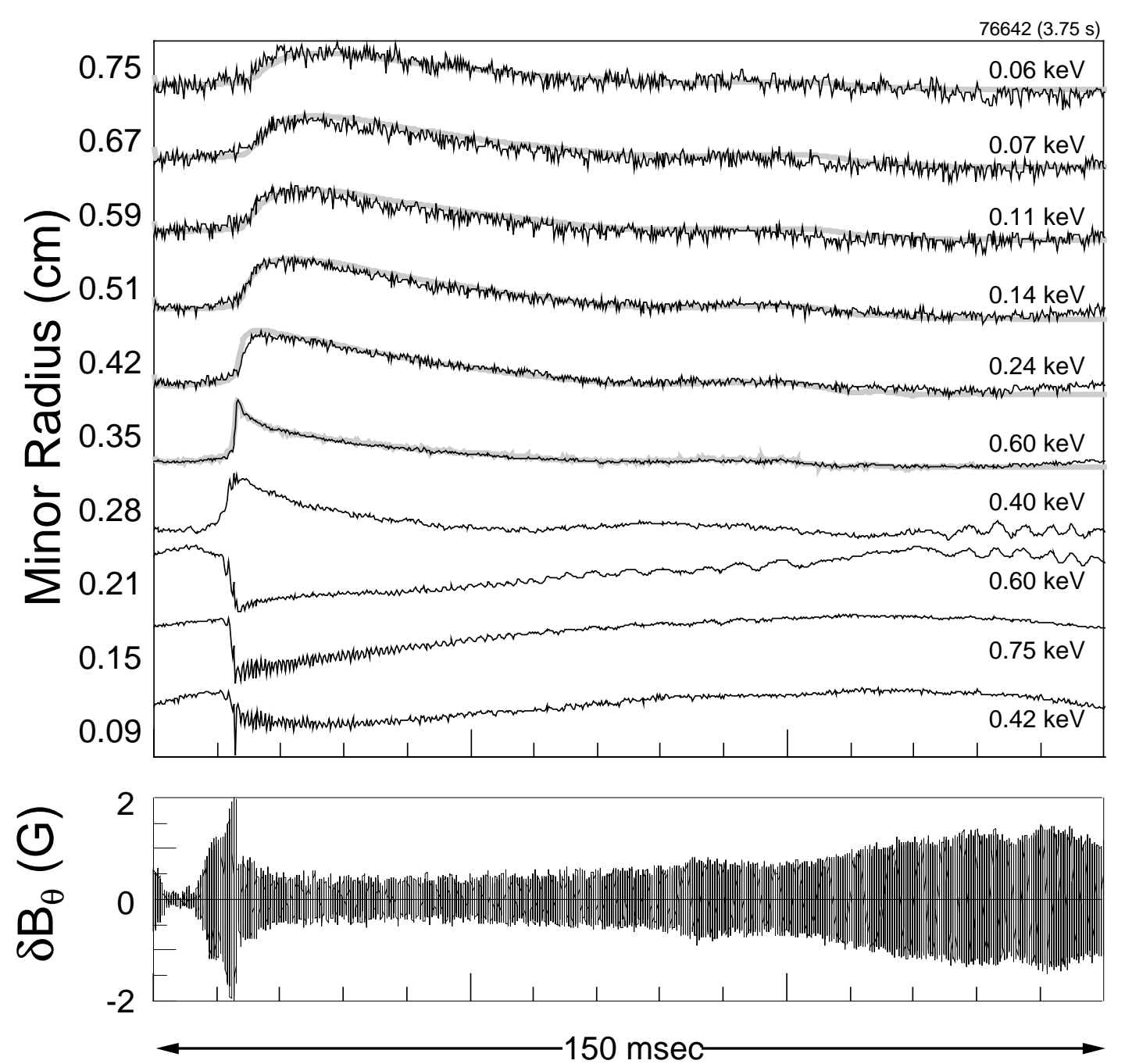
Figure 19
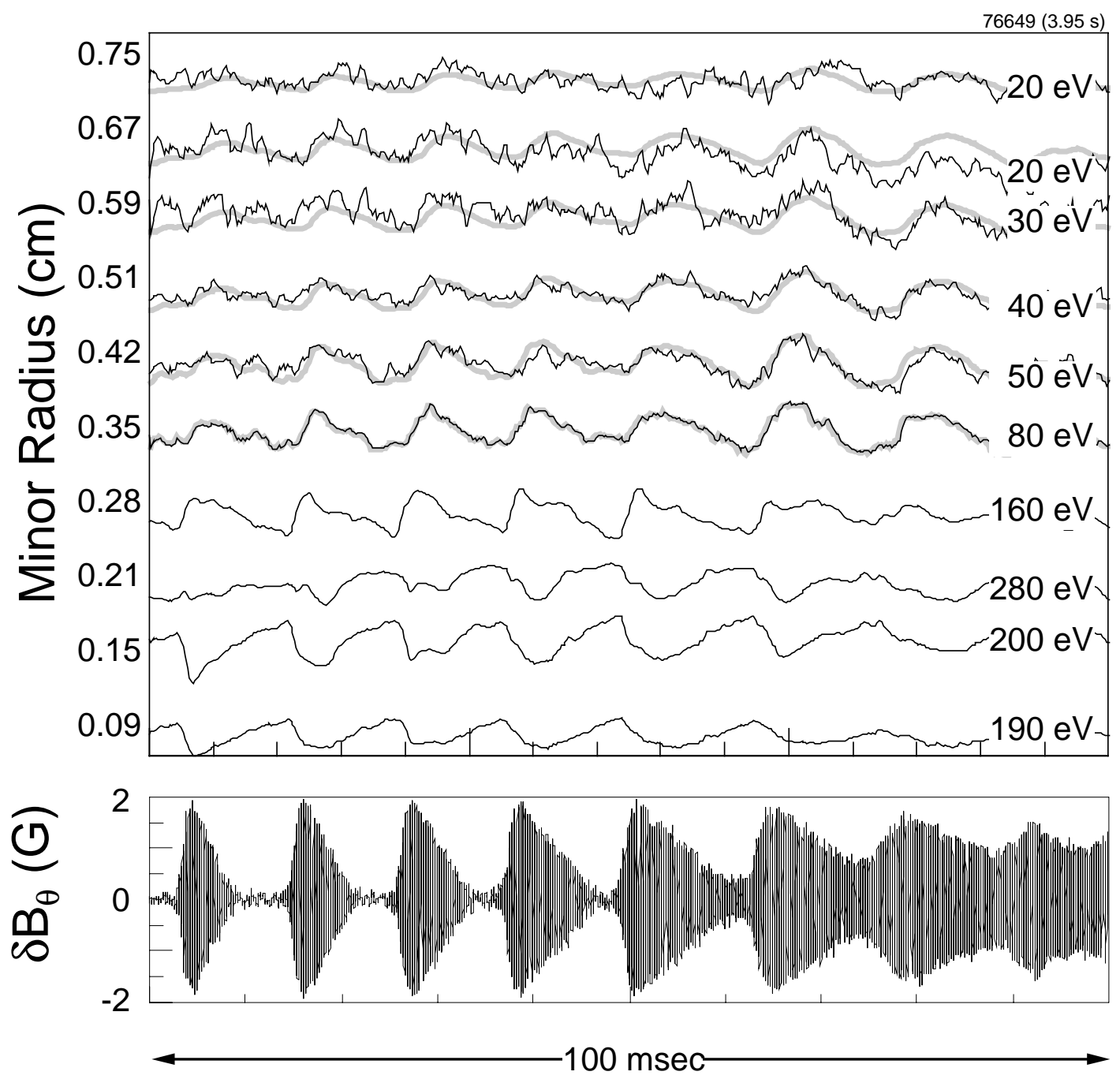
The Princeton Plasma Physics Laboratory is operated by Princeton University under contract with the U.S. Department of Energy.

\author{
Information Services \\ Princeton Plasma Physics Laboratory \\ P.O. Box 451 \\ Princeton, NJ 08543
}

Phone: 609-243-2750

Fax: 609-243-2751

e-mail: pppl_info@pppl.gov

Internet Address: http://www.pppl.gov 